\title{
Administración del riesgo temporal de liquidez, asociado a los Ilamados al margen, dentro del negocio de comercialización del café verde en Colombia
}

Carlos Armando Mejía Vega*

* Docente, Universidad Externado de Colombia. Magíster en Finanzas, Observatorio de Economía y Operaciones Numéricas.carlos.mejia@uexternado.edu.co

Fecha de recepción: 24 de febrero de 2015.

Fecha de aceptación: 02 de abril de 2015.

Forma de citar

Mejía Vega, C. A. (2015). Administración del riesgo temporal de liquidez, asociado a los llamados al margen dentro del negocio de comercialización del café verde en Colombia. ODEON, 9, pp. 173-231. DOI: http://dx.doi.org/10.18601/17941113.n9.05 



\section{Introducción}

Como lo señaló en algún momento el economista británico John Maynard Keynes $^{1}$, en la mayoría de empresas productoras o exportadoras de commodities, en particular las de carácter agrícola, el valor del Capital Operativo de Trabajo es mayor que el del Activo Fijo ${ }^{2}$. Lo anterior implica que, si una firma desea maximizar su valor, esta deba concentrarse primordialmente en la dinámica del inventario y de las cuentas por cobrar (es decir, en los procesos de producción, compra, venta y recaudo) así como en sus riesgos asociados. Al revisar lo anterior, y siguiendo una vez más a Keynes, se evidencian al menos dos tipos de riesgos ${ }^{3}$ : de un lado, uno de mercado, producto de las fluctuaciones que puede tener el precio en el mercado físico internacional entre los momentos de producción (o de compra) y de venta del commodity y, del otro, de liquidez, asociado a las necesidades de corto plazo para financiar todo el proceso hasta que se realiza el recaudo de la venta ${ }^{4}$ (Fantacci, Marcuzzo y Sanfilippo, 2010).

Con base en lo anterior, resulta fundamental para este tipo de empresas el poder cubrir de la mejor forma posible los dos riesgos mencionados con anterioridad. En ese sentido, para mitigar el primero, señala Keynes, las empresas acuden a los mercados de contratos futuros sobre commodities ${ }^{5}$ en los que toman la posición

1 John Maynard Keynes fue un importante especulador en los mercados de contratos futuros sobre commodities desde 1920 hasta 1938. Se mantuvo fuertemente activo en el algodón, pero también en otros como plomo, estaño, cobre, caucho, trigo y azúcar (Fantacci, Marcuzzo y Sanfilippo, 2010).

2 Respecto a esto, hay que decir que Keynes hablaba en realidad de capital circulante y capital fijo, siguiendo la tradición de David Ricardo y de John Stuart Mill, que llegó a él de la mano de su maestro Alfred Marshall. Sin embargo, al leer detenidamente sus trabajos, resulta evidente que corresponden a los modernos conceptos del capital operativo de trabajo y de activos fijos.

3 En el presente artículo no se revisa el riesgo de tasa de cambio, dejando su análisis para futuros trabajos.

4 En la actualidad, si el ciclo operativo (rotación de inventarios y de cartera) es superior a la rotación de proveedores, las firmas se enfrentan a un riesgo de liquidez. Para cubrirlo, como lo señala Keynes, las empresas acuden a los bancos (Fantacci, Marcuzzo y Sanfilippo, 2010). Lo anterior representa una importante labor para muchas áreas de tesorería.

5 De acuerdo con Hull, los contratos futuros se definen como un acuerdo entre dos partes para comprar o vender un activo, llamado subyacente, en un momento en el futuro llamado vencimiento $T$, por un precio determinado llamado Precio Futuro $F_{p, T}$ el cual se establece en el momento $t=p$, pero que se diferencian de los contratos forward en cuanto estos se negocian en mercados estructurados por bolsas organizadas que implican condiciones estandarizadas (Hull, 2009).

PP. $173-231 \cdot N .^{\circ} 9 / 2015$ 
contraria a la que tienen en el mercado físico internacional. Sin embargo, este economista, al igual que muchas empresas productoras o exportadoras de commodities agrícolas en la actualidad, no advirtió algo fundamental: el uso de este tipo de instrumentos financieros conlleva un riesgo temporal de liquidez que puede limitar o entorpecer toda la estrategia de cobertura si no se tiene en cuenta. En efecto, el incumplimiento de los llamados al margen implica que la posición en el contrato futuro se cierre antes de que se haga lo mismo en el mercado físico internacional, dejando descubierta nuevamente la posición.

Así, el presente artículo busca sintetizar los principales avances alcanzados en la tesis de grado "Administración del riesgo temporal de liquidez, asociados a los llamados al margen dentro del negocio de comercialización del café verde" ", la cual buscaba desarrollar algunos esquemas ${ }^{7}$ que permitieran administrar el mencionado riesgo, tomando un commodity de carácter agrícola insignia para Colombia como es el café verde de la especie vegetal arábica (en adelante café verde arábica), el cual históricamente han exportado las empresas colombianas, producto de la ventaja comparativa que existe en dicho país para producirlo ${ }^{8}$.

Con base en lo anterior, en la primera parte del artículo se describirá el Contrato "C" de Café de la IntercontinentalExchange Inc. (en adelante ICE) y su importancia en el mercado físico internacional de café verde arábica. La segunda parte desarrollará el esquema de cobertura básico que permite mitigar el riesgo de precio a través de este contrato futuro y con el que se pueda evidenciar en toda su dimensión el riesgo temporal de liquidez asociado a los llamados al margen que se les pueden llegar a presentar a las empresas exportadoras que hacen uso de

6 Tesis de Grado para optar al título de Magíster en Finanzas de la Universidad Externado de Colombia (2014). Con el fin de que el lector pueda remitirse a la fuentes que permitieron la elaboración de este trabajo de grado, se mantendrán las citas del trabajo original, salvo en aquellos extractos propios del documento, los cuales se citarán como Mejía (2014).

7 La inspiración para este trabajado de grado surge de una revisión del capítulo 25 de Wilmott (2007) en el que señalaba que uno de los riesgos que la mayoría consideran incubribles era precisamente el originado por los llamados al margen, el cual no había recibido mucho interés en el mundo académico. Este autor, sin embargo, piensa que, por el contrario, sí es un riesgo que se puede gestionar, y propone una solución a partir de la metodología de los chashmetrics, sin profundizar mucho la misma o en alguna otra.

8 El Coffea es uno de los principales géneros de la familia de plantas conocidas como Rubiáceas. Una de las especies vegetales más importantes es la Coffea arábica linneo. Con base en lo anterior, al fruto que se obtiene del mismo, una vez pasa por los procesos de recolección, beneficio y trilla, se conoce como café arábica o arábiga verde y es el commodity que exporta Colombia en el mercado físico internacional.

PP. $173-231 \cdot N .{ }^{\circ} 9 / 2015$ 
este tipo de estrategias ${ }^{9}$. En la tercera parte, se describirá el modelo de proyección para el Precio Futuro en el corto plazo. En la cuarta parte, se desarrollarán los dos esquemas de administración mediante la constitución de un fondo preventivo $\mathrm{y}$, finalmente, en la última parte, se presentan las principales conclusiones y extensiones al presente artículo con el fin de incentivar el desarrollo de futuros trabajos al respecto.

\section{EI Contrato "C" de Café de la ICE y su importancia dentro del mercado internacional del café verde arábica}

El contrato futuro que más se utiliza para realizar coberturas que permitan mitigar el riesgo de precio, dentro del mercado físico internacional del café verde arábica, se conoce como Contrato " $\mathrm{C}$ " de Café (en adelante Contrato "C"). Este instrumento es actualmente estructurado por la bolsa ICE ${ }^{10}$ y se negocia en el mercado norteamericano de Nueva York, conocido como IntercontinentalExchange, Inc. Futures U.S. (en adelante ICE Futures U.S.). Las características generales del mismo se muestran a continuación (IntercontinentalExchange, Inc., 2013) (tabla 1):

9 Existen muchos esquemas de cobertura para un exportador, no solo a través de contratos futuros, sino de otros instrumentos como las opciones. Sin embargo, dada la baja cultura financiera de las empresas en Colombia, se optó por el esquema más sencillo, el cual, no obstante, permite evidenciar con total claridad la dinámica de los llamados al margen.

10 La Coffee, Sugar and Cocoa Exchange (CS\&C) fue la primera en estructurar el Contrato "C" en el año 1882. En el año 1998 pasaría a ser parte de la New York Board of Trade (NYвOT), у más tarde, durante finales de 2006 y comienzos de 2007, pasó a formar parte de la IntercontinentalExchange, Inc. (ICE) (2013). 
Tabla 1. Especificaciones del Contrato "C"

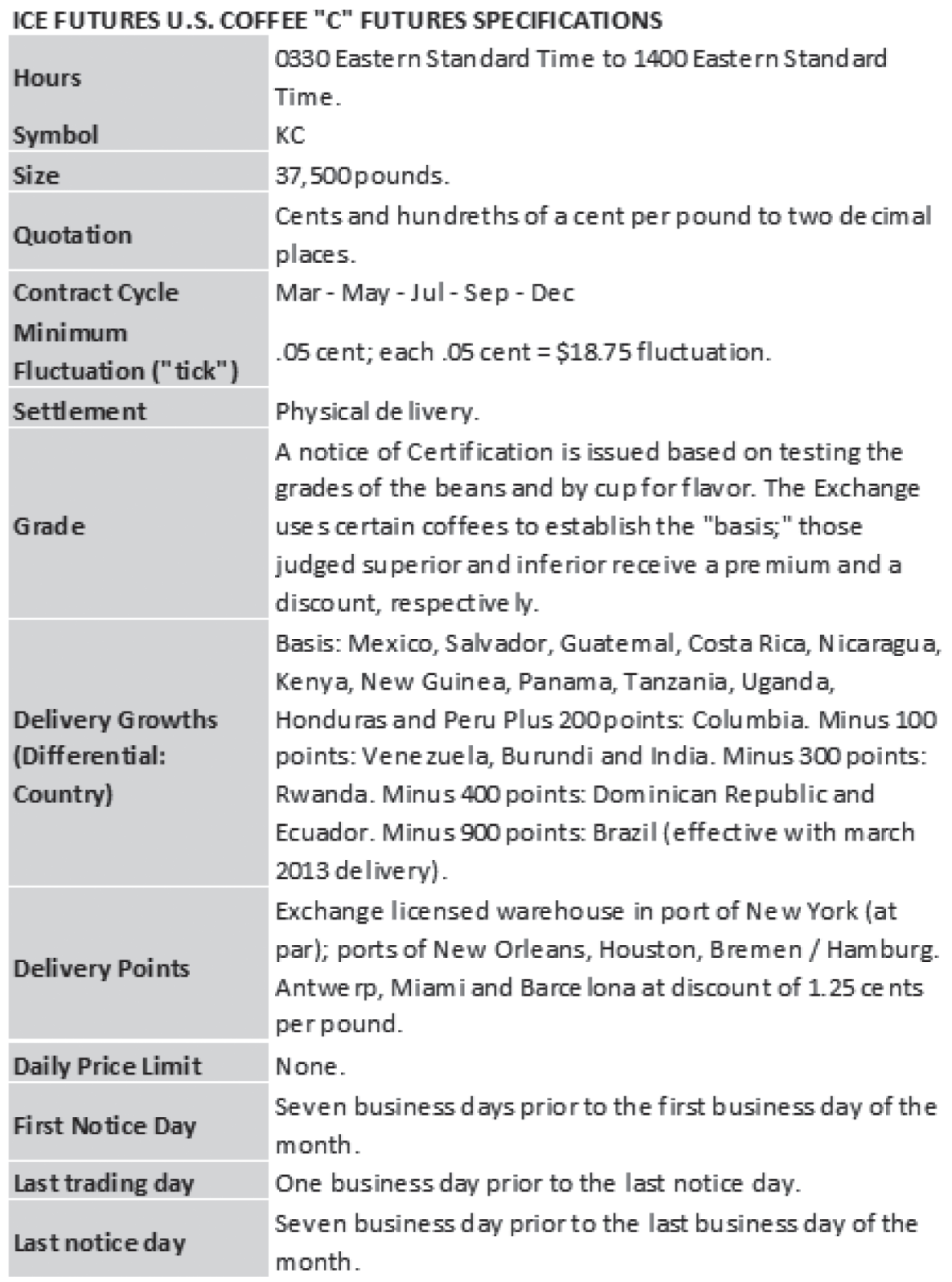

Tomado de Mejía (2014). 
Lo anterior se debe a que el Precio Futuro $F_{t, T}^{11}$ del Contrato "C" cumple un papel fundamental dentro de la negociación que se lleva a cabo en el mercado físico internacional. En efecto, en un mundo ausente de dicho contrato futuro y producto de la dinámica de las exportaciones, exportadore ${ }^{12}$ e importadore ${ }^{13}$, en el instante $t=c$, celebrarían un Contrato Forward al precio vigente en el mercado ${ }^{14}$, el cual se denotará como Precio Forward $J_{c, E}$, para entregar una determinada cantidad de algún tipo de café verde ${ }^{15}$ en el tiempo $t=E$. Como se aprecia en el gráfico 1 , el riesgo de precio se presenta entre el momento que el exportador fija el Precio Forward con el importador y el momento $t=f$ en el que compra el café pergamino seco (CPS $)^{16}$ a algún tercero en el país, tomando como referencia el Precio Forward $J_{f, E}($ Mejía, 2014).

11 A lo largo del artículo se utiliza la terminología de Helfrich (2009) pero, dada la dinámica de los llamados al margen, se manejará todo en tiempo discreto.

12 Corresponde a las unidades productivas de los países que tienen alguna ventaja comparativa en la producción del café verde arábica y que, por ende, lo exportan, tales como Brasil, Vietnam, Colombia e Indonesia (IntercontinentalExchange, Inc., 2013).

13 Corresponde a las unidades productivas de los países que no tienen alguna ventaja comparativa en la producción del café verde arábica y que, por ende, lo importan, tales como la Unión Europea, Estados Unidos y Japón (IntercontinentalExchange, Inc., 2013).

14 Siguiendo a Helfrich (2009), el proceso que sigue el Precio Forward se denota como.

15 Hay miles de tipos de café verde. La Organización Internacional del Café los clasifica en tres grandes grupos: i) Suaves colombianos; ii) Naturales brasileros y iii) Otros (Organización Internacional del Café, 2013).

16 Dentro del contexto del café arábica, se conoce como CPS al café que aun no ha pasado por el proceso de trilla. Siguiendo a Rosas (1993, citado en EAFIT, 2007), en Colombia, un exportador de café verde tiene la obligación de "disponer de (por lo menos) una trilladora para garantizar el procesamiento del producto que comercializa", por lo que "su papel no se puede restringir al de la intermediación". 
Gráfico 1. Riesgo de precio para un exportador en el mercado físico internacional de café verde arábica en un mundo ausente del Contrato " $\mathrm{C}$ "

Precio forward

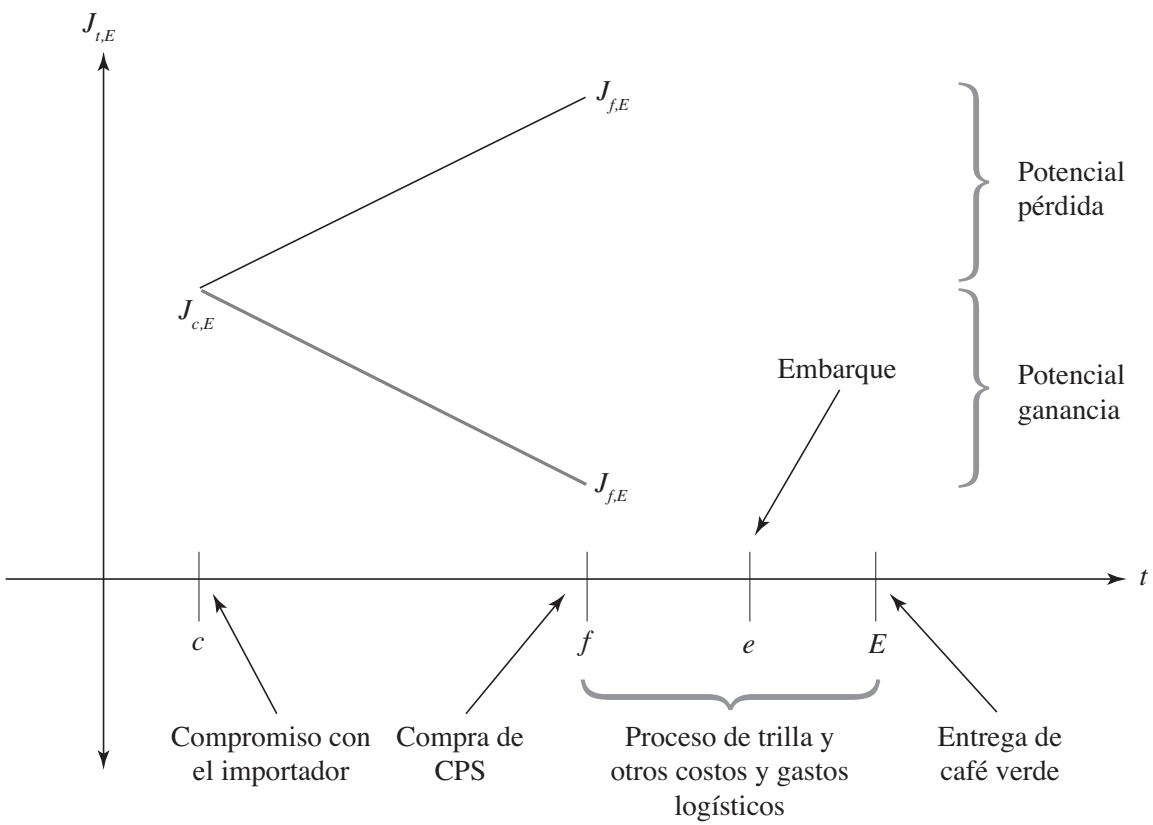

Tomado de Mejía (2014).

Sin embargo, con la introducción del Contrato "C", nace el primer supuesto esencial y tácito dentro de la comercialización de café verde, el cual señala que dado que con los contratos futuros se negocia un commodity estandarizado por la ICE, y con los contratos forward se negocia otro pero con algún grado de diferenciación, entonces la única diferencia a través del tiempo $t$, tal que $E \leq T^{17}$, entre los Precios Futuros y los Precios Forward corresponde a la discrepancia por la calidad del café, llamada diferencial, como se aprecia a continuación (International Trade Centre, 2011):

$$
D_{t}=J_{t, E}-F_{t, T}
$$

Donde:

17 Se debe recordar que en realidad existirán tantos Contratos "C" como vencimientos existan. En ese orden de ideas, el mercado presume que el Precio Futuro asociado para cada Precio Forward, corresponde al del contrato futuro con el vencimiento más cercano al del contrato forward. 
$D_{t}$ : diferencial a través del tiempo $t$

Dado lo anterior, es posible partir el Precio Forward en dos partes: i) el Precio Futuro y ii) lo que se llamará un Diferencial Forward, que se constituye en una variable que se fija con el importador (se toma una posición larga o corta en el mismo) en algún momento del tiempo, producto de la calidad del café verde por encima de la del café estándar que sirve de subyacente al Contrato "C", y también de las habilidades del exportador e importador a la hora de comprar y de vender. De esta forma, al sumarle el Precio Futuro se obtiene el Precio Forward (International Trade Centre, 2011):

$$
\begin{aligned}
& D_{t, E}=J_{t, E}-F_{t, T} \\
& D_{t, E}+F_{t, T}=J_{t, E}
\end{aligned}
$$

Donde:

$D_{t, E}:$ diferencial forward a través del tiempo $t$

Con base en lo anterior, ahora el proceso de negociación entre el exportador y el importador se divide en dos momentos (International Trade Centre, 2011) ${ }^{18}$ :

- En el momento $t=c$, el exportador celebra con el importador un contrato forward para fijar con el mismo un diferencial de venta $D_{c, E}$ para entregarle $k$

18 Lo anterior supone que en el mercado físico internacional se "cree" que el Contrato "C" refleja correctamente la dinámica de la oferta y la demanda de la porción estándar del café verde arábica. Este hecho puede resultar controversial, en la medida que, como señalaba el mismo Keynes, la única forma en que este tipo de mercados de contratos futuros gocen de liquidez, es con la existencia de agentes especuladores que le apuntan a que el Precio Futuro se mueva en las direcciones opuestas a las que buscan cubrir exportadores e importadores respectivamente. En ese sentido, si estos agentes tienen la capacidad de incidir sobre el mercado (a diferencia de la industria), podrían generar burbujas especulativas sobre dichos precios. El problema surge si se tiene presente que esos Precios Futuros, en última instancia, serán la base con la que exportadores e importadores compran o venden a los productores y consumidores finales respectivamente, y si se encuentran inflados, producirían un efecto económico negativo (o positivo) sobre los mismos que nada tiene que ver con la dinámica propia de la oferta y la demanda. Es un caso más, dentro del contexto del capitalismo financiero, que muestra cómo las finanzas están incidiendo cada vez más sobre la dinámica real. 
cantidad de algún tipo de café verde en el momento de tiempo $t=E$, tal que $c<E$.

- En el momento $t=f$, el exportador fija con el importador el Contrato "C" al Precio Futuro $F_{f, T}$ vigente en el ICE Futures U.S., tal que $c<f<E$, con el fin de determinar el Precio Forward de venta final.

- En el momento $t=E$, el exportador entrega al importador la cantidad de café verde y el importador le paga $D_{c, E}+F_{f, T}=J_{c, E}$

Como se aprecia en el gráfico 2, el riesgo de precio también se divide ahora en dos partes: i) un riesgo de diferencial, similar al del precio del primer escenario (gráfica 1), que se materializa cuando el exportador compra el cPs y ii) un riesgo asociado a las subidas o bajadas del Contrato "C" entre la compra del café y la fijación del Precio Futuro con el importador (Mejía, 2014).

Gráfico 2. Riesgo de precio para un exportador en el mercado físico internacional de café verde arábica en presencia de Contrato "C"19

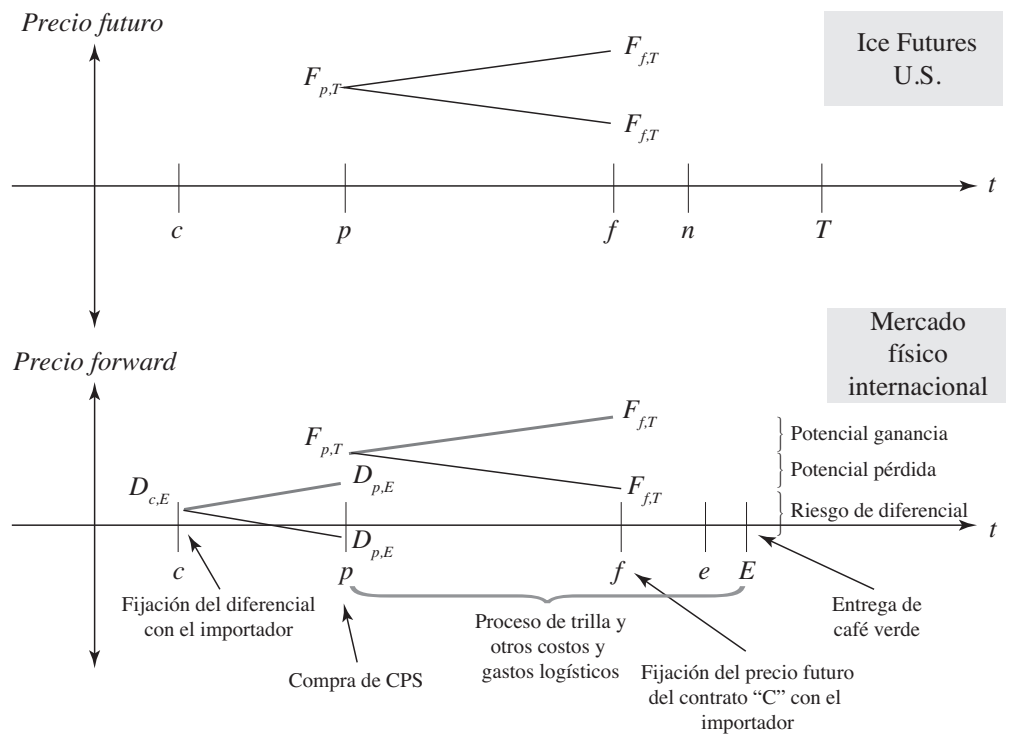

Adaptado de Mejía (2014).

19 Como el lector podrá apreciar en el gráfico 2, el diferencial (a diferencia del Precio Futuro del Contrato "C") puede adquirir valores negativos. En ese caso, se constituiría en un "castigo" frente al café estándar que sirve de subyacente al contrato futuro. Si se desea ver los "premios" o "castigos" que, por ejemplo, impone la ICE por procedencia del café, el lector puede remitirse a la tabla 1 de esta artículo (IntercontinentalExchange, Inc., 2013). 


\section{El esquema de cobertura y el riesgo temporal de los llamados al margen}

\subsection{El esquema de cobertura}

Con esto en mente, el esquema de cobertura que, se asume, toman los exportadores de café verde, con el que resuelven el riesgo de precio visto en la sección 1, es el siguiente (Mejía, 2014):

- En el momento $t=c$, el exportador fija con el importador un diferencial de venta $D_{c, E}$ para entregarle $k$ cantidad de café verde en el momento de tiempo $t=E$, tal que $c<E$.

- Ya con el diferencial de venta, las cantidades y la fecha estimada de embarque, el exportador ajusta su logística de compra de café diaria para vincular el cPs requerido con el pedido del importador. Así, cuando va a comprar en el momento $t=p$ :

- Se revisa la cotización de la Primera Posición Relevante del contrato "C" con vencimiento en $t=T_{1}^{20}$ cuyo primer día de noticias $t=n$ sea inferior a $t=E$ y se encuentre alrededor de la fecha esperada de embarque establecida con el importador en $t=e$.

- Con esa referencia, entra a buscar la cantidad de CPS en el mercado local y al momento de comprarlo ofrece un precio equivalente a $J_{p, E}{ }^{21}=F_{p, T}+$ $D_{c, E}$, donde $D_{p, E}$ denota el diferencial de compra y en el que se busca que $D_{c, E}>D_{p, E}$

- Toma una posición corta en una serie de Contratos "C" en el ICE Futures U.S. por el equivalente a la cantidad de CPS que se requiere ${ }^{22}$, al precio futuro $F_{p, T^{*}}$

20 Se conoce como la Primera Posición Relevante al contrato cuyo First Notice Day y su vencimiento se denotarán como $T_{1}$. En ocasiones, estos dos coinciden hasta los últimos días previos al First Notice Day, pues la mayoría de inversionistas se comienzan a concentrar más en el siguiente contrato.

$21 \quad$ A este precio se le deben descontar los costos y gastos en que debe incurrir el exportador desde que compra el crs hasta que entrega el café verde, en cuanto los precios forward son ex-dock. Sin embargo, en el presente trabajo no se realiza esta ardua labor en cuanto se escapa del objetivo del mismo. 22 Lo anterior es difícil de lograr, no solo para el café verde, sino también para cualquier commodity. En efecto, dado que un contrato futuro tiene un determinado tamaño, muchas veces no 
- En el momento $t=f$, tal que $f \leq n(\operatorname{si} n<e)$ o $f \leq e(\text { si } e<n)^{23}$ el exportador termina de fijar con el importador las condiciones de la venta:

- Se revisa la cotización de la Primera Posición Relevante del Contrato "C" con vencimiento en $t=T_{1}$ cuyo primer día de noticias $t=n$ sea inferior a $t=E$ y se encuentre alrededor de la fecha esperada de embarque establecida con el importador en $t=e$.

- Con esta referencia, fija con el importador un precio equivalente a $F_{f, T}$, de tal forma que el precio final sea $J_{f, E}=F_{f, T}+D_{c, E}$.

- Toma una posición larga por la misma cantidad de contratos futuros en el ICE Futures U.S. para cerrar la posición al precio Futuro $F_{f, T}$.

Al final, en el momento $t=f$ se tiene el siguiente balance:

- Como se vio en la sección 1, el exportador tendrá una pérdida o ganancia en el mercado físico internacional por la diferencia entre $J_{p, E} \mathrm{y} J_{f, E}$.

- Por otro lado, este se obligó a vender a un precio $F_{p, T}$ y luego se obligó a comprar la misma cantidad por otro precio $F_{f, T}$, por lo que tendrá una pérdida o ganancia por la diferencia entre $F_{p, T}$ y $F_{f, T}$.

- Dado que $J_{p, E}=F_{p, T}+D_{p, E}$ y $J_{f, E}=F_{f, T}+D_{c, E}$, entonces la ganancia o pérdida neta es la siguiente:

$$
\begin{gathered}
\text { Resultado }=\left(J_{f . E}-J_{p . E}\right)+\left(F_{p, T}-F_{f, T}\right) \\
\text { Resultado }=\left[\left(F_{f, T}+D_{c, E}\right)-\left(F_{p, T}+D_{p, E}\right)\right]+\left(F_{p, T}-F_{f, T}\right)
\end{gathered}
$$

se requiere de una unidad entera del derivado para realizar la cobertura dejando lo que se conoce coloquialmente como "colas". La gestión de las mismas es una de las labores más importantes que realiza un área de riesgo en materia de cobertura de riesgo de precio. Este problema no se presenta con instrumentos отс (Over the Counter) al estar hechos "a la medida" (Hull, 2009).

23. Lo anterior significa que si la fecha de embarque $t=e$ se encuentra en un horizonte superior al First Notice Day, el punto máximo para fijar el precio con el importador es el First Notice Day porque, de lo contrario, podría exponerse al riesgo de tener que recibir café por parte de la ICE. Si por alguna razón no se fijara el precio, el exportador debería cerrar la posición en esa fecha y tomar una nueva posición corta en el contrato siguiente (conocido como Roll-Over). Esta posibilidad no altera en absoluto la forma de administrar los llamados al margen. Por otro lado, si la fecha de embarque $t=e$ se encuentra en un horizonte inferior al First Notice Day, el punto máximo para fijar el precio con el importador es esa fecha ya que, de lo contrario, no podría embarcarse el café verde.

PP. $173-231 \cdot N .^{\circ} 9 / 2015$ 


$$
\text { Resultado }=F_{f, T}+D_{c, E}-F_{p, T}-D_{p, E}+F_{p, T}-F_{f, T}
$$

$$
\text { Resultado }=D_{c, E}-D_{p, E}
$$

El anterior esquema se muestra en el gráfico 3.

\section{Gráfico 3. Esquema de cobertura con la Primera Posición} Relevante del Contrato "C"

\section{Precio futuro}

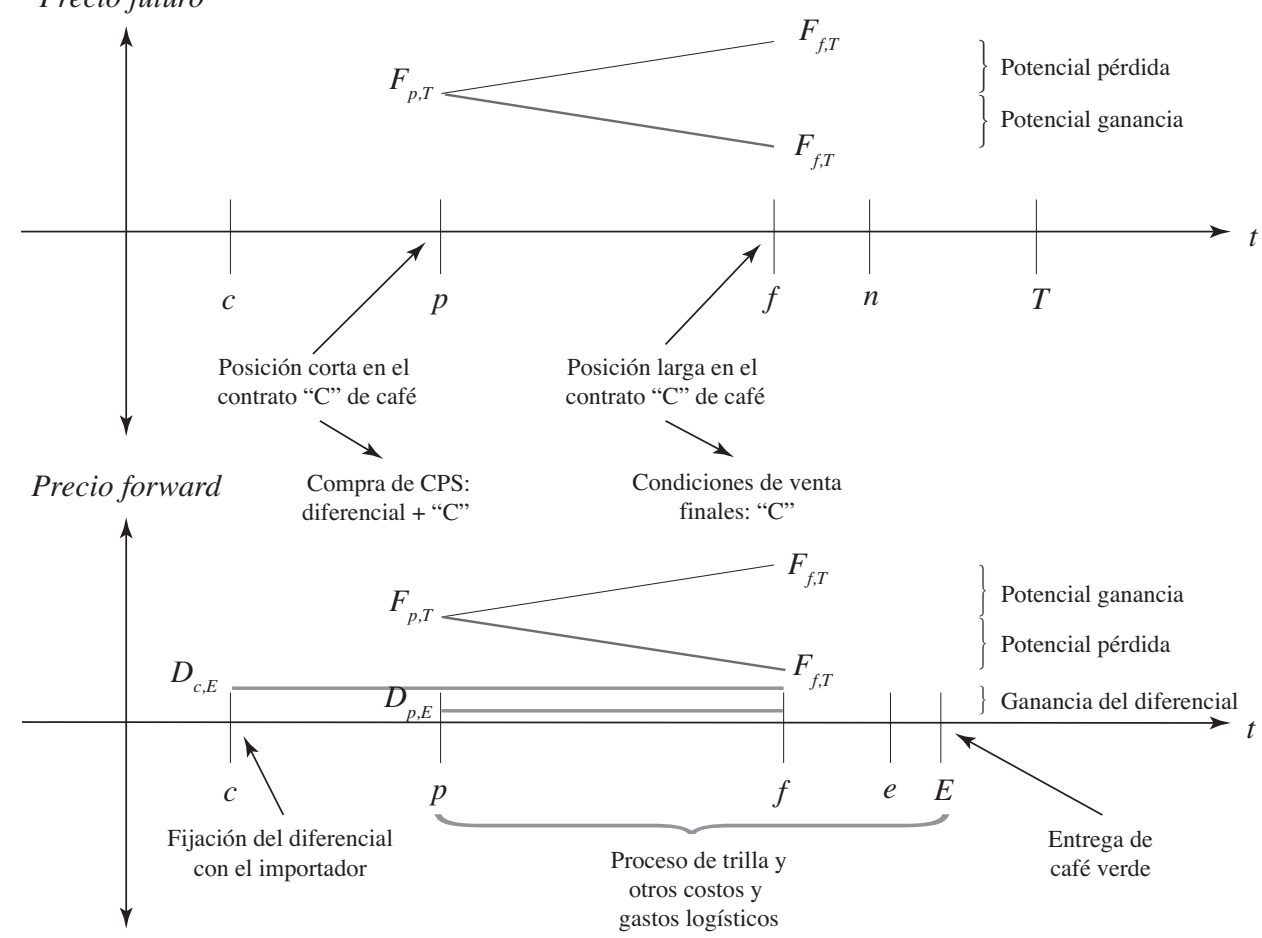

Tomado de Mejía (2014).

De lo anterior, se concluye que mediante este esquema de cobertura, los exportadores lograrían mitigar una porción importante del riesgo de precio (la porción estándar), haciendo que el nivel del Contrato " $\mathrm{C}$ " se vuelva irrelevante para ellos, y permitiendo que la rentabilidad y el riesgo del negocio dependan exclusivamente de los niveles del diferencial de compra y de venta, lo que se 
constituye nuevamente en otra modalidad del llamado riesgo de base (Mejía, $2014)^{24}$.

\subsubsection{Escenario para el ejercicio del backtesting}

Con el fin de evaluar la aplicabilidad de las estrategias de administración del riesgo temporal de liquidez asociadas a los llamados al margen que se van a presentar más adelante, se propone el uso del procedimiento conocido como backtesting. Con base en lo anterior, el escenario ideal para poner a prueba los esquemas es un periodo en el que el Precio Futuro del Contrato " $C$ " haya presentado una tendencia alcista de manera permanentemente ${ }^{25}$. En ese sentido, un periodo relativamente reciente de subida para los sucesivos contratos futuros se presentó a finales del 2007 hasta comienzos del $2011^{26}$. Con base en lo anterior, el periodo de estudio se concentrará entre el 19 de mayo al 23 de agosto de 2010. Se propone entonces un escenario en el que un exportador de café colombiano, posterior a una negociación del diferencial con un importador, entra a comprar CPS con un intermediario, tomando como referencia el Contrato "C" con vencimiento en septiembre de 2010, para posteriormente fijar el precio de venta final:

El 19 de mayo de 2010 el exportador, por temas de la ciclicidad de la cosecha, tiene la posibilidad de comenzar a comprar un CPs bastante especial. Si bien lo lógico sería realizar las negociaciones con la Primera Posición Relevante del Contrato "C" de ese momento, la cual correspondía a la posición de julio, al momento de revisar su relación compras/ventas, el exportador se percata que cuenta en su inventario con las cantidades necesarias para suplir los embarques programados para junio.

24 Se invita al lector a tratar de diseñar esquemas que permitan cubrir la dinámica del Diferencial Forward, ya que si bien representa una porción pequeña del Precio Forward, lo cierto es que su fluctuación puede ser representativa y, bajo el modelo actual, no existen esquemas claros para cubrir su riesgo.

25 El concepto de tendencia se puede revisar al menos desde dos perspectivas: i) desde el análisis técnico y ii) desde el punto de vista econométrico. Sin embargo, en este contexto se refiere a la sucesión de Precios Futuros cada vez más altos.

26 Al momento de redactarse este artículo, los sucesivos Precios Futuros de los Contratos "C" experimentaron, desde comienzos del 2014 hasta finales de ese mismo año, una importante tendencia alcista, producto de la incertidumbre de la cosecha de Brasil ante posibles escenarios de sequía. Sin embargo, la tesis de grado que sirve de base para este artículo se elaboró bajo el otro escenario y, por ello, es que se mantiene el mismo.

PP. $173-231 \cdot N . \circ 9 / 2015$ 
Sin embargo, a la hora de revisar julio se da cuenta que tiene una entrega por 486 sacos de $70 \mathrm{~kg}^{27}$, de ese café verde especial para el 10 de julio con un importador, a un diferencial de venta de 40 ctvos./b, y que no cuenta con el mismo para suplirlo. De esta forma, dado que el embarque debería ser los primeros días de julio, ya no tendría sentido utilizar la posición de julio y sí la de septiembre, cuyo First Notice Day era el 23 de agosto (Mejía, 2014).

Se tiene entonces la siguiente estrategia de coberturas (Mejía, 2014):

- El exportador compra el 19 de mayo de 2010 cPs a un intermediario:

- Se revisa la cotización del Contrato "C" de Café con vencimiento en septiembre el cual al cierre de esa fecha fue de $133,80^{28}$.

- Con esa referencia, entra a buscar los 45.358 kilos de CPS $^{29}$ en el mercado local y al momento de comprarlo ofrece un precio equivalente a $J_{p, E}=133,80+25=158,80$.

- Toma una posición corta en 2 contratos " $C$ " de Café ${ }^{30}$ en el ICE Futures U.S. al precio futuro $F_{p, T}=133,80$.

- Entre el 19 de mayo y el 7 de julio al exportador le entra ese cPs a su inventario, trilla los 45.358 kilos de CPS, obtiene los sacos de $70 \mathrm{~kg}$ de café verde e incurre en todos los otros costos y gastos logísticos para entregar el commodity.

- El exportador fija el 7 de julio de 2010 el precio final con el importador:

- Se revisa la cotización del Contrato "C" de Café con vencimiento en esa fecha el cual al cierre fue de $163,05^{31}$.

- Con esta referencia, fija con el importador un precio final de $J_{f, E}=163,05$ $+40=203,05$.

- Toma una posición larga en 2 Contratos “C” de Café en el ICE Futures U.S. al precio futuro $F_{p, T}=163,05$.

27 En Colombia es común negociar cantidades expresadas en sacos de $70 \mathrm{~kg}$.

28 Se asume que el cPs se compró con el cierre del Precio Futuro de la posición de septiembre del Contrato "C". En la práctica, sin embargo, puede tomarse cualquier precio a lo largo de la cotización diaria.

29 En términos generales, para obtener un saco de $70 \mathrm{~kg}$ de café verde colombiano (conocido como café excelso, el cual corresponde al café verde apto para exportación al cumplir con las políticas de calidad que exige la Federación Nacional de Cafeteros) se requiere de $93 \mathrm{~kg}$ de CPS.

30 Hay que recordar que un Contrato "C" equivale a 37.500 libras de café verde.

31 Se asume que la fijación del precio final se realiza con el cierre del Precio Futuro de la posición de septiembre del Contrato "C". En la práctica, no obstante, puede tomarse cualquier precio a lo largo de la cotización diaria. 
El balance al final es el siguiente:

$$
\begin{gathered}
\text { Resultado }=[163,05+40-(133,80+25)]+(133,80-163,05) \\
\text { Resultado }=163,05+40-133,80-25+133,80-163,05 \\
\text { Resultado }=40-25 \\
\text { Resultado }=15
\end{gathered}
$$

\subsection{El riesgo temporal de liquidez asociado a los llamados al margen}

\subsubsection{Generalidades}

Ahora bien, entre $t=p$ y $t=f$, la posición corta en el Contrato " $\mathrm{C}$ " que tiene el exportador cambiará de valor a través del tiempo dados los nuevos Precios Futuros de los siguientes contratos con igual vencimiento en $T_{1}$. Siguiendo a Helfrich (2009), denotaremos el valor de mercado de esta posición como $\Lambda$. De esta forma, en el momento $t=p$, el valor del contrato futuro será $\Lambda(p ; 0, T)=0$ y de ahí en adelante cambiaría de la siguiente forma (IntercontinentalExchange, Inc., 2013):

$$
\Lambda(p ; t, T)=\left(\frac{F_{p, T}-F_{t, T}}{\gamma}\right) m x^{32}
$$

Donde:

$\gamma:$ tick del Contrato "C"

$\omega:$ tamaño del Contrato "C"

$$
\begin{gathered}
\gamma * \omega: \text { multiplicador del Contrato "C" } \\
x: \text { número de contratos futuros }
\end{gathered}
$$

32 Esta es la forma como la ICE oficialmente calcula las ganancias o las pérdidas en una posición (IntercontinentalExchange, Inc., 2013). 
Ahora bien, una última particularidad de los contratos futuros es que a estos, a diferencia de los contratos forward, se les aplica una práctica conocida como marked to market de manera diaria. Lo anterior significa que las ganancias (pérdidas) se reciben (pagan) diariamente, llevando el valor del contrato para las dos posiciones nuevamente a cero (Helfrich, 2009). Para llevar a cabo esta práctica, la ICE exige a quienes hacen uso de este tipo de contratos futuros (en este caso los exportadores de café verde) que tengan una cuenta de margen con un broker. La cantidad mínima que se debe depositar en esta cuenta al momento de tomar la posición corta, conocida como el margen inicial, dependerá del tamaño y de la volatilidad histórica del mismo (Helfrich, 2009) y se denotará como $\mathrm{M}_{\mathrm{I}}$.

Así, al final de cada día de transacción en $t+\Delta t$, la cuenta se ajusta para reflejar el marked to market: el efectivo entrará a la cuenta si la posición es ganadora $\left(\mathrm{F}_{t}>\right.$ $\mathrm{F}_{t+\Delta t}$ ) o saldrá si es perdedora (cuando $\mathrm{F}_{t}<\mathrm{F}_{t+\Delta t}$ ). Sin embargo, para garantizar que la cuenta de margen no adquiera un valor negativo en ningún momento, la ICE fija una segunda cantidad conocida como margen de mantenimiento, que se denotará como $\mathrm{M}_{\mathrm{m}}$. Así, si el valor de la cuenta de margen cae por debajo de este nivel, el exportador recibe lo que se conoce como un llamado al margen, el cual implica que debe depositar recursos adicionales a los comprometidos inicialmente hasta llegar nuevamente al margen inicial el día inmediatamente siguiente ${ }^{33}(\mathrm{Hull}, 2009)$.

La situación anterior implica que mientras el exportador no cierre la posición cuando fija el precio de venta final con el importador, las subidas en el Precio Futuro pueden generar necesidades de caja adicionales a las comprometidas al momento de tomar la posición inicial. Si estas no se cumplen, el broker se verá en la necesidad de cerrar la posición forzosamente dejando la contraparte en el mercado físico internacional descubierta (IntercontinentalExchange, Inc., 2013). Lo anterior genera un riesgo temporal de liquidez.

\subsubsection{Escenario para el ejercicio del backtesting}

Retomando el escenario del backtesting, se recuerda que entre el 19 de mayo y el 7 de julio de 2010 el exportador mantuvo un portafolio con dos posiciones cortas de Contratos "C". De esta forma, desde el momento en el que se deposita el margen inicial de US\$3.115 por contrato y, dado el margen de mantenimiento

\footnotetext{
33 Si bien el llamado al margen se debe responder al día siguiente, para efectos de modelaje lo importante es que este se origina en el día del cierre del Precio Futuro.
} 
de US\$2.515 por contrato (Bloomberg, s. f.), a través del tiempo se presentarían sucesivos llamados al margen, como se muestra en la tabla 2:

Tabla 2. Dinámica de los llamados al margen para el escenario de backtesting

\begin{tabular}{|c|c|c|c|c|c|c|}
\hline Dias & $\begin{array}{l}\text { Precio } \\
\text { Futuro }\end{array}$ & $\begin{array}{c}\text { Ganancia } \\
\text { (pérdida) } \\
\text { Diaria }\end{array}$ & $\begin{array}{c}\text { Ganancia } \\
\text { (pérdida) } \\
\text { Acumulada }\end{array}$ & $\begin{array}{c}\text { Balance } \\
\text { Cuenta } \\
\text { Margen }\end{array}$ & $\begin{array}{l}\text { Lamado al } \\
\text { margen }\end{array}$ & $\begin{array}{c}\text { Balance } \\
\text { Cuenta } \\
\text { Margen } \\
\text { Final }\end{array}$ \\
\hline $19 / 05 / 2010$ & 133,80 & & & 6.230 & & 6.230 \\
\hline $20 / 05 / 2010$ & 132,95 & 638 & 638 & 6.868 & - & 6.868 \\
\hline $21 / 05 / 2010$ & 133,70 & (563) & 75 & 6.305 & - & 6.305 \\
\hline $24 / 05 / 2010$ & 133,65 & 37 & 113 & 6.343 & - & 6.343 \\
\hline $25 / 05 / 2010$ & 134,25 & $(450)$ & (337) & 5.893 & - & 5.893 \\
\hline $26 / 05 / 2010$ & 135,25 & $(750)$ & $(1.087)$ & 5.143 & - & 5.143 \\
\hline $27 / 05 / 2010$ & 136,30 & $(788)$ & $(1.875)$ & 4.355 & 1.875 & 6.230 \\
\hline $28 / 05 / 2010$ & 135,80 & 375 & $(1.500)$ & 6.605 & - & 6.605 \\
\hline $01 / 06 / 2010$ & 138,05 & $(1.688)$ & (3.188) & 4.918 & 1.313 & 6.230 \\
\hline $02 / 06 / 2010$ & 136,90 & 863 & $(2.325)$ & 7.093 & - & 7.093 \\
\hline $03 / 06 / 2010$ & 137,05 & $(113)$ & $(2.438)$ & 6.980 & - & 6.980 \\
\hline $04 / 06 / 2010$ & 135,30 & 1.313 & $(1.125)$ & 8.293 & - & 8.293 \\
\hline $07 / 06 / 2010$ & 134,60 & 525 & $(600)$ & 8.818 & - & 8.818 \\
\hline $08 / 06 / 2010$ & 135,10 & $(375)$ & $(975)$ & 8.443 & - & 8.443 \\
\hline $09 / 06 / 2010$ & 136,05 & $(713)$ & $(1.688)$ & 7.730 & - & 7.730 \\
\hline $10 / 06 / 2010$ & 138,55 & $(1.875)$ & $(3.563)$ & 5.855 & - & 5.855 \\
\hline $11 / 06 / 2010$ & 146,10 & $(5.662)$ & $(9.225)$ & 193 & 6.037 & 6.230 \\
\hline $14 / 06 / 2010$ & 151,60 & $(4.125)$ & $(13.350)$ & 2.105 & 4.125 & 6.230 \\
\hline $15 / 06 / 2010$ & 159,95 & $(6.263)$ & $(19.613)$ & $(32)$ & 6.263 & 6.230 \\
\hline $16 / 06 / 2010$ & 159,60 & 262 & $(19.350)$ & 6.493 & - & 6.493 \\
\hline $17 / 06 / 2010$ & 157,80 & 1.350 & $(18.000)$ & 7.842 & - & 7.842 \\
\hline $18 / 06 / 2010$ & 162,10 & $(3.225)$ & $(21.225)$ & 4.618 & 1.613 & 6.230 \\
\hline $21 / 06 / 2010$ & 160,80 & 975 & $(20.250)$ & 7.205 & - & 7.205 \\
\hline $22 / 06 / 2010$ & 160,75 & 38 & $(20.213)$ & 7.243 & - & 7.243 \\
\hline $23 / 06 / 2010$ & 160,50 & 188 & $(20.025)$ & 7.430 & - & 7.430 \\
\hline $24 / 06 / 2010$ & 168,75 & $(6.188)$ & $(26.213)$ & 1.243 & 4.988 & 6.230 \\
\hline $25 / 06 / 2010$ & 168,90 & $(113)$ & $(26.325)$ & 6.118 & - & 6.118 \\
\hline $28 / 06 / 2010$ & 168,10 & 600 & $(25.725)$ & 6.718 & - & 6.718 \\
\hline $29 / 06 / 2010$ & 163,15 & 3.712 & $(22.013)$ & 10.430 & - & 10.430 \\
\hline $30 / 06 / 2010$ & 165,85 & $(2.025)$ & $(24.038)$ & 8.405 & - & 8.405 \\
\hline $01 / 07 / 2010$ & 168,25 & $(1.800)$ & $(25.838)$ & 6.605 & - & 6.605 \\
\hline $02 / 07 / 2010$ & 164,30 & 2.962 & $(22.875)$ & 9.567 & - & 9.567 \\
\hline $06 / 07 / 2010$ & 157,65 & 4.988 & $(17.888)$ & 14.555 & - & 14.555 \\
\hline $07 / 07 / 2010$ & 163,05 & $(4.050)$ & $(21.938)$ & 10.505 & - & 10.505 \\
\hline
\end{tabular}

Tomado de Mejía (2014).

PP. $173-231$ • N. ${ }^{\circ} 9 / 2015$ 
Nótese que si bien la pérdida acumulada es de US\$21.938, esta se compensaría con la ganancia en el mercado físico internacional. Sin embargo, los llamados al margen acumulados ascienden a US $\$ 26.214$ y corresponden a necesidades inmediatas de caja que debe suplir el exportador en tanto no cierre la posición o, de lo contrario, toda la lógica de cobertura de precio vista en la sección 2.1 se puede perder.

\subsubsection{Dinámica de la cuenta de margen}

A partir de la tabla 2, y al revisar la evolución de la columna número 4, se evidencia que su dinámica se puede representar a través de una Ecuación en Diferencias de Primer Orden, lo que nos lleva a la proposición 1 (Mejía, 2014):

Proposición 1. Una forma de representar la variación en la cuenta de margen para un portafolio de $x$ contratos " $C$ ", en los que se tiene una posición corta, a través del tiempo es la siguiente:

$$
\Delta M_{t}=\left(\frac{F_{t, T}-F_{t+\Delta t, T}}{\gamma}\right) m x
$$

Donde:

$\Delta M_{t}=M_{t+\Delta t}-M_{t}$ : variación en la cuenta de margen

Al resolver la Ecuación en Diferencias por el método de la iteración (anexo 1) se tiene que (Mejía, 2014):

$$
M_{t}=\left(\frac{F_{0, T}-F_{t, T}}{\gamma}\right) m x+M_{I} x
$$

\section{Modelo de Proyección del Precio Futuro del Contrato "C" 34}

De lo visto en la sección 2 resulta evidente que la dinámica del Precio Futuro del Contrato "C", que hasta ahora y producto del esquema de cobertura de precio se

34 El presente modelo se presentará inicialmente en tiempo continuo, pero una vez se cuente con la dinámica para el Precio Futuro del Contrato "C" se procederá a discretizarlo nuevamente. 
había vuelto irrelevante, vuelve a tomar importancia, por lo que resulta fundamental su modelación a través del tiempo.

\subsection{Variables}

\subsubsection{El Precio Spot Estándar}

Se presume la existencia de un segundo mercado físico internacional en el que se celebran contratos spot ${ }^{35}$ del café verde estándar que sirve como subyacente al Contrato "C". En este mercado se cumpliría con los tres supuestos del principio de competencia perfecta y, por ende, también con la ley de un solo precio, el cual se denota como Precio Spot Estándar $S(t)$ y quedaría determinado por las fuerzas económicas de la oferta y la demanda (Pindyck y Rubinfeld, 2006).

\subsubsection{La Convenience Yield Neta}

Se presume la existencia de una tasa continua conocida como Convenience Yield Bruta y, la cual simboliza el flujo de servicios que le corresponden al dueño del café verde físico en comparación con quien ostenta un Contrato "C" para entrega del mismo en el futuro (Brennan y Schwartz, 1985). Sin embargo, dado que el almacenamiento de este commodity implica también una serie de costos y gastos, conocidos como Storage Costs (Hull, 2009), se trabajará entonces con la Convenience Yield Neta, la cual se define como la diferencia entre la Convenience Yield Bruta y los Storage Costs, y que se denotará como $\delta$ (Helfrich, 2009).

\subsubsection{La Tasa Libre de Riesgo}

Se presume la existencia de una Tasa Libre de Riesgo a la que los agentes del mercado se pueden endeudar o pueden invertir sus excedentes y que se denotará como $r_{f}($ Hull, 2009).

\footnotetext{
35 De acuerdo con Hull (2009), un contrato spot es un acuerdo entre dos partes para comprar y vender un commodity hoy, es decir, con entrega inmediata.
} 


\subsubsection{Supuestos para el ICE Futures U.S.}

Adicional a los tres supuestos expuestos con anterioridad, se presume que en el ICE Futures U.S. se cumplen las siguientes condiciones (Helfrich, 2009):

- Toda la información disponible no tiene costo alguno para todos y cada uno de los participantes.

- No existe costo alguno para transar el Contrato "C".

- No existen impuestos gravados a las utilidades que se puedan obtener.

- Los arbitrajistas pueden tomar ventaja de las oportunidades de arbitraje.

\subsection{La relación entre el Precio Spot Estándar y el Precio Futuro en un mismo momento del tiempo}

Dadas las condiciones planteadas anteriormente, y siguiendo a Hull (2009) y Helfrich (2009), el Precio Futuro del Contrato "C" con vencimiento en $T$, debe obedecer a la siguiente relación para todo momento del tiempo $t$ :

$$
F(t, T)=S(t) e^{c c(T-t)}
$$

Donde:

cc: Costo of Carry $y^{36}$

Sin embargo, siguiendo a Helfrich (2009) se opta por la descomposición del Cost of Carry, en cuanto permite una clara separación de influencia de cada una de las variables vistas con anterioridad (anexo 2):

$$
F(t, T)=S(t) e^{\left(r_{f}-\delta\right)(T-t)}
$$

Esta última expresión será de gran ayuda para desarrollar el modelo de proyección del Precio Futuro del Contrato "C", una vez se tenga alguna dinámica para el Precio Spot Estándar, la Convenience Yield Neta y la Tasa Libre de Riesgo.

\footnotetext{
36 Por Cost of Carry se entiende el costo total de tener un inventario del commodity. Con base en lo anterior, se calcula tomando los Storage Costs y los Gastos Financieros (representados en este caso a través de la Tasa Libre de Riesgo) y restándole la Convenience Yield Bruta (Hull, 2009).
} 


\subsection{Proyección del Precio Spot Estándar}

Dado el horizonte de proyección que se requiere de corto plazo, en cuanto interesa exclusivamente la dinámica del Precio Futuro de la Primera Posición Relevante del Contrato "C", se planteará una modificación al modelo de Gibson y Schwartz (1990) y, más específicamente, al segundo de Schwartz (1997) en el que la tendencia constante que sigue el Retorno Simple Neto Instantáneo, disminuida en la Convenience Yield Neta Instantánea contante $^{37}$, es de corto plazo:

$$
\begin{array}{r}
\frac{d S(t)}{S(t)}=\left(\mu_{C P}-\delta\right) d t+\sigma_{S} d W(t) \\
d S(t)=\left(\mu_{C P}-\delta\right) S(t) d t+\sigma_{S} S(t) d W(t)
\end{array}
$$

Donde:

$\mu_{C P}$ : media de corto plazo del Retorno Simple Neto instantáneo del Precio Spot Estándar

$\sigma_{S}:$ desviación estándar instantánea de corto plazo del Precio Spot Estándar $d W(t)$ : diferencial del Proceso de Wiener Estándar

$W(t)$ : Proceso de Wiener Estándar bajo la medida de probabilidad $\mathbb{P}^{38}$

$$
W(0)=0
$$

$W(t)$ es continuo para todo $t \geq 0$

$W(t)$ se distribuye normalmente bajo la distribución de probabilidad $\mathbb{P}$ con media 0 y desviación estándar 1

$W(t)$ tiene incrementos $\mathrm{dW}(\mathrm{t})$ independientes y normalmente distribuidos

37 Nótese que dentro de este modelo ya se está definiendo la dinámica para la Convenience Yield Neta. Dado el horizonte de tiempo que se requiere, se puede asumir como constante.

38 Existen innumerables textos que explican la construcción del Proceso Wiener Estándar y, por ende, no se detalla a lo largo del presente texto. 


\subsection{Proyección del Precio Futuro del Contrato "C"}

Una vez se cuenta con una expresión para la evolución temporal de la Convenience Yield Neta y del Precio Spot Estándar y, dada la relación vista en (4), se puede proceder a encontrar una expresión para la evolución temporal del Precio Futuro del Contrato "C". Asumiendo que la Tasa Libre de Riesgo es también constante, se puede encontrar la dinámica del Precio Futuro aplicando el lema de Ito (anexo 3) (Hull, 2009):

$$
d F(t, T)=F(t, T)\left(\mu_{C P}-r_{f}\right) d t+\sigma_{S} F(t, T) d W(t)
$$

De esta forma, al igual que el Precio Spot Estándar, el Precio Futuro seguiría un Movimiento Browniano Geométrico en el que, sin embargo, el componente de tendencia es $\left(\mu_{C P}-r_{f}\right)$ en vez de $\left(\mu_{C P}-\delta\right)^{39}$. Se procede a resolverlo nuevamente a través del lema de Ito (Hull, 2009) (anexo 4):

$$
F(t, T)=F(0, T) e^{\left[\left(\mu_{C P}-r_{f}\right)-\frac{1}{2} \sigma_{S}^{2}\right] t+\sigma_{S} \int_{0}^{t} d W(z)}
$$

\subsection{Modelo para realizar las simulaciones en el escenario de backtesting ${ }^{40}$}

Una vez se cuenta con una expresión que describe la evolución temporal del Precio Futuro del Contrato "C", el siguiente paso es utilizarla para realizar simulaciones de MonteCarlo. Para ello, se procede a discretizar la ecuación 11 de la siguiente forma:

$$
\begin{gathered}
F(t, T)=F(0, T) e^{\left[\left(\mu_{C P}-r_{f}\right)-\frac{1}{2} \sigma^{2}\right] t+\sigma \int_{0}^{t} d W(z)} \\
\text { Discretizando } \\
F_{t+\Delta t, T}=F_{t, T} e^{\left[\left(\mu_{C P}-r_{f}\right)-\frac{1}{2} \sigma^{2}\right] \Delta t+\sigma \varphi \sqrt{\Delta t}}
\end{gathered}
$$

39 Lo anterior refleja lo mencionado respecto a la Convenience Yield. En efecto, quien ostenta un Contrato "C" no puede beneficiarse del flujo de servicios que garantiza la tenencia del commodity físico. De ahí la razón de que desaparezca de la expresión para el Precio Futuro.

$40 \quad$ La calibración de este modelo se puede apreciar en el anexo 5. 


$$
\begin{gathered}
\Delta t=\frac{T}{\tau} \text { es el incremento en el tiempo } \\
T \text { es el horizonte de la proyección }
\end{gathered}
$$

$\tau$ es el número de pasos en los que se divide la proyección

$$
\begin{gathered}
\varphi \text { es una variable que sigue una distribución normal } \\
\text { con media } 0 \text { y desviación estándar } 1
\end{gathered}
$$

Esta última expresión será la que se utilizará para los procesos de simulación por el método de MonteCarlo más adelante

\section{El esquema de administración}

El esquema que se explora para administrar el riesgo temporal de liquidez asociado a los llamados al margen se inspira en su dinámica básica, es decir, en el hecho de que una vez se genere el mismo se debe depositar una suma en la cuenta de margen que la lleve nuevamente al nivel del margen inicial. En ese sentido, resulta razonable pensar que una forma de administrar el mencionado riesgo es, en vez de esperar a que el broker exija dicha suma, que se cuente de antemano con tales recursos a través de un fondo preventivo (Taulli, 2011) ${ }^{41}$. Lo anterior da origen a dos posibles esquemas: i) uno estático y ii) uno condicionado en el que se va recalculando el monto del fondo a medida que transcurre el tiempo (Mejía, 2014).

\subsection{Esquema estático de administración}

El primer paso bajo este esquema de administración es el de calcular el Precio Futuro que daría origen al llamado al margen en el futuro y que se constituye como una barrera (Mejía, 2014):

\footnotetext{
41 Esta idea la propone Taulli (2011) al afirmar que una forma de lidiar con los llamados al margen es depositando más recursos en la cuenta. Sin embargo, al igual que Wilmott (2007) con la metodología del chashmetrics, este no profundiza en la mejor manera de hacerlo.
} 
Esquema I - Paso 1. En el momento en el que se toma una posición corta en $x$ Contratos "C" que buscan cubrir una compra de CPS en el momento $t=p$ a un Precio Futuro $F_{p, T}$, se procede a calcular el Precio Futuro barrera $F_{B_{1,1}{ }^{\prime} T_{1}}$, que originaría el primer llamado al margen:

$$
\text { Dado que } F_{p, T}-F_{B_{1,1}, T_{1}}<0^{43}
$$

Se busca que

$$
\begin{gathered}
x M_{t} \leq x M_{M} \\
M_{t} \leq M_{M}
\end{gathered}
$$

Dada la relación de la ecuación 6

$$
\begin{gathered}
M_{t}=\left(\frac{F_{p, T_{1}}-F_{B_{1,1}, T_{1}}}{\gamma}\right) m+M_{I} \\
\text { Entonces } \\
\left(\frac{F_{p, T_{1}}-F_{B_{1,1}, T_{1}}}{\gamma}\right) m+M_{I} \leq M_{M} \\
\left(\frac{F_{p, T_{1}}-F_{B_{1,1}, T_{1}}}{\gamma}\right) m \leq M_{M}-M_{I}
\end{gathered}
$$

42 Los sufijos 1,1 se explicarán más adelante. Por el momento, se le pide al lector que los tome como referencia.

43 Dado que la posición del exportador en el contrato futuro es corta, los llamados al margen solo se producirán ante subidas en el Precio Futuro. 


$$
\begin{gathered}
\left(\frac{F_{p, T_{1}}-F_{B_{1,1}, T_{1}}}{\gamma}\right) \leq \frac{M_{M}-M_{I}}{m} \\
F_{p, T_{1}}-F_{B_{1,1}, T_{1}} \leq\left(\frac{M_{M}-M_{I}}{m}\right) \gamma \\
-F_{B_{1,1}, T_{1}} \leq\left(\frac{M_{M}-M_{I}}{m}\right) \gamma-F_{p, T_{1}} \\
F_{B_{1,1}, T_{1}} \geq F_{p, T_{1}}-\left(\frac{M_{M}-M_{I}}{m}\right) \gamma
\end{gathered}
$$

Dado que el tick del Contrato "C" es de 0,05 ctvos/lb (tabla 1), y que lo que se tiene es una desigualdad, entonces se aproxima hacia arriba tomando el siguiente Precio Futuro (Mejía, 2014):

$$
F_{B_{1,1}, T_{1}}=\left\langle\text { Redondear }\left\{\frac{\left[F_{p, T_{1}}-\left(\frac{M_{M}-M_{I}}{m}\right) \gamma\right]}{\gamma}\right\}\right\rangle * \gamma+\gamma^{44}
$$

$\mathrm{Al}$ aplicar lo anterior al ejercicio de backtesting, se tiene el siguiente cálculo (Mejía, 2014):

44 El dividir por el tick, luego redondear y multiplicar nuevamente por el mismo es un ajuste para que el Precio Futuro siempre fluctúe en esa cantidad. Por otro lado, el sumarle el tick al final se utiliza para aproximarlo al precio de más arriba ya que, de un lado, la transformación anterior lo redondea al valor de más abajo y, del otro, es la única forma de que el Precio futuro toque la barrera. 


$$
\begin{gathered}
F_{B_{1,1}, T_{1}}=\left\langle\text { Redondear }\left\{\frac{133,80-\left(\frac{2.500-3.115}{18,75}\right) 0,05}{0,05}\right\}\right\rangle * 0,05+0,05 \\
F_{B_{1,1}, T_{1}}=134,65^{45}
\end{gathered}
$$

Una vez se cuenta con el Precio Futuro que originaría el primer llamado al margen, el siguiente paso consiste en realizar una proyección del Precio Futuro del Contrato "C" con el fin de identificar una posible trayectoria $i=1$ que seguirá este a través del tiempo y así estimar el posible momento en el que se produzca el llamado al margen ${ }^{46}$. Siguiendo a Wilmott (2007), esta proyección se llevará a cabo a partir de simulaciones de Monte Carlo con base en la ecuación 12 y tomando como punto inicial de la proyección el Precio Futuro $F_{p, T_{1}}$ sobre el horizonte de tiempo requerido. Se tiene así el segundo paso (Mejía, 2014):

Esquema I - Paso 2. En el momento $t=p$ se procede a proyectar una posible trayectoria $i=1$ del Precio Futuro del Contrato "C", desde $t=p$ hasta $t=T_{1}$. Una vez se tiene, se procede a revisar si ese camino toca el Precio Futuro $F_{B_{1,1}, T}$, el cual origina el primer llamado al margen, en algún punto.

45 El lector notará que si se aproximara hacia abajo tomando el Precio Futuro $F_{h_{1}, T_{1}}=134,60$, no se tocaría el margen de mantenimiento planteado de 2.500 como se muestra a continuación:

$* 2$

$$
M_{I}-\left(\frac{F_{p, T}-F_{B_{1}, T_{1}}}{\gamma}\right) m x=2.500-\left(\frac{133,80-134,60}{0,05}\right) 18,75
$$

$M_{I}-\left(\frac{F_{p, T}-F_{B_{1}, T_{1}}}{\gamma}\right) m x=2.515$

46 Ya desde este punto se puede apreciar que la solución propuesta consiste en dividir el problema en dos partes: i) determinar el Precio Futuro Barrera y avaluar si el Precio Futuro lo toca en algún punto, y ii) calcular el llamado al margen en ese momento del tiempo con el Precio Futuro Efectivo. Más adelante, esta particularidad permitirá construir, con ayuda de la ecuación 12, dos distribuciones que al unirse (proceso conocido como convolución) permiten obtener un valor del fondo preventivo solventando algunas limitaciones de los dos esquemas de administración propuestos. 
Al realizar una simulación de Monte Carlo, dentro del escenario de backtesting, y tomando como Precio Futuro inicial $F_{p, T_{1}}=133,80$ sepuede apreciar que en algún punto en adelante se toca la barrera $F_{B_{1,1}, T_{1}}$ (gráfico 4).

Gráfico 4. Primer Precio Futuro que genera el llamado al margen en el escenario de backtesting

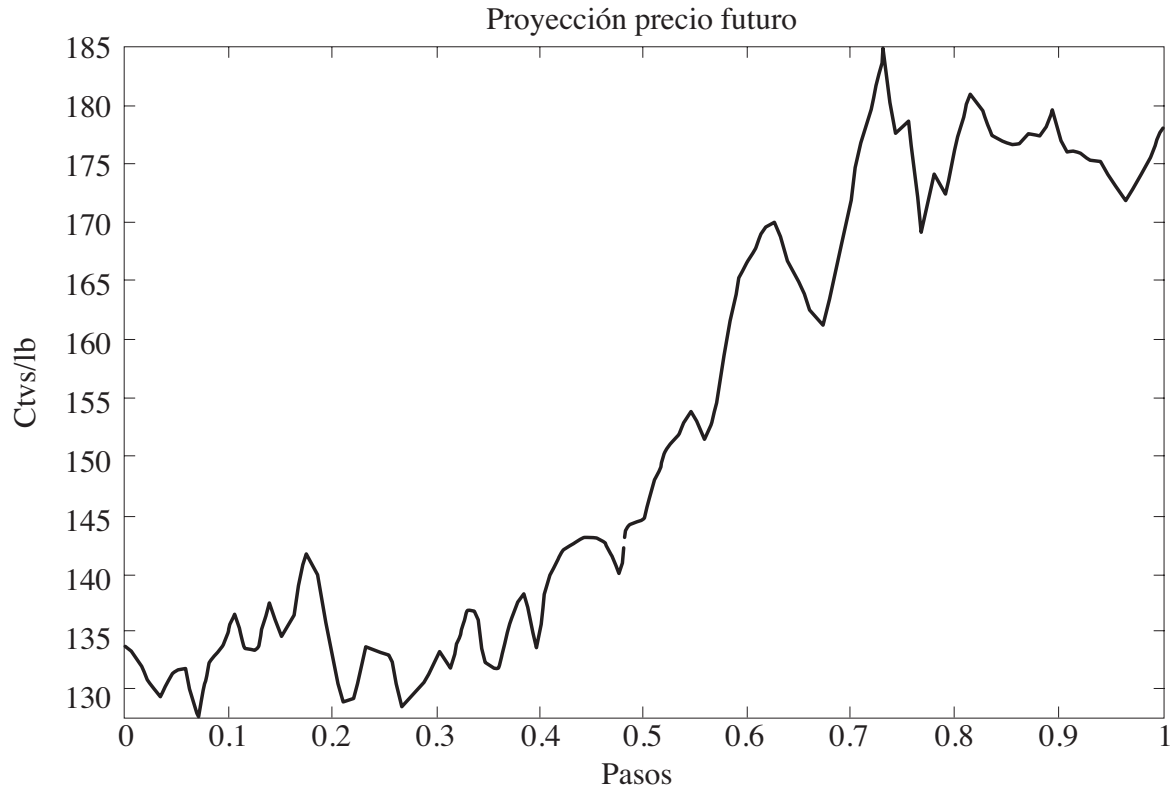

Precio Futuro proyectado desde el 19 de mayo hasta el 20 de septiembre, dado un Precio Futuro $F(0, T)=133,80$. Proyección bajo el modelo de Gibson y Schwartz (1990), el segundo de Schwartz (1997) o de Brennan y Schwartz (1985) con Media diaria de $\mu=0,3804 \%$, Desviación Estándar diaria de $\sigma=2,2563 \%$ y Tasa Libre de Riesgo $r_{f}=0,0019 \%$ (anexo 5) (Mejía, 2014).

Ahora bien, en caso de presentarse una situación de llamados al margen en algún momento del tiempo $t=h_{1,1}$, aún se pueden seguir presentando otros más adelante, por lo que se debe proceder a calcular el siguiente Precio Futuro que originaría el próximo llamado al margen. Con base en lo anterior, se debe tener en cuenta la diferencia entre el Precio Futuro barrera $F_{B_{1,1}, T_{1}}$ que origina el llamado al margen en el tiempo $t=h_{1,1}$ y el Precio Futuro efectivo que eventualmente lo genera $F_{h_{1,1}, T_{1}}$. Es con base en este último que se realiza el siguiente cálculo (Mejía, 2014). 
Esquema I - Paso 3. Dados los $j^{47}$ llamados al margen que se han presentado para la trayectoria $i=1$, y teniendo el último Precio Futuro efectivo $F_{h_{i, j} T_{1}}$ que lo generó en el momento de tiempo $t=h_{i, j}$, se puede calcular el siguiente Precio Futuro barrera $F_{B_{i, j+1}, T_{1}}$, que originaría el próximo llamado al margen.

$$
\text { Dado que } F_{h_{i, j}, T_{1}}-F_{B_{i, j+1}, T_{1}}<0^{48}
$$

Se busca que

$$
\begin{gathered}
M_{t} \leq M_{M} \\
\text { Retomando } \\
M_{t}=\left(\frac{F_{h_{i, j}, T_{1}}-F_{B_{i, j+1}, T_{1}}}{\gamma}\right) m+M_{I}
\end{gathered}
$$

Entonces

$$
\left(\frac{F_{h_{i, j}, T_{1}}-F_{B_{i, j+1}, T_{1}}}{\gamma}\right) m+M_{I} \leq M_{M}
$$

$47 j=0, \ldots$, es una variable que indica el número de veces que se han producido llamados al margen a la hora de calcular el Precio Futuro barrera.

48 Nótese que si $i=1$ (trayectoria 1 de la simulación de Monte Carlo) y $j=0$ (no se han producido llamados al margen), entonces $F_{h_{i, j}, T_{1}}=F_{h_{1,0}, T_{1}}=F_{p, T_{1}}$ y $F_{B_{i, j+1}, T_{1}}=F_{B_{1,1}, T_{1}}$ llegando al paso 1
nuevamente. 


$$
\begin{gathered}
\left(\frac{F_{h_{i, j}, T_{1}}-F_{B_{i, j+1}, T_{1}}}{\gamma}\right) m \leq M_{M}-M_{I} \\
\left(\frac{F_{h_{i, j}, T_{1}}-F_{B_{i, j+1}, T_{1}}}{\gamma}\right) \leq \frac{M_{M}-M_{I}}{m} \\
F_{h_{i, j}, T_{1}}-F_{B_{i, j+1}, T_{1}} \leq\left(\frac{M_{M}-M_{I}}{m}\right) \gamma \\
-F_{B_{i, j+1}, T_{1}} \leq\left(\frac{M_{M}-M_{I}}{m}\right) \gamma-F_{h_{i, j}, T_{1}} \\
F_{B_{i, j+1}, T_{1}} \geq F_{h_{i, j}, T_{1}}-\left(\frac{M_{M}-M_{I}}{m}\right) \gamma
\end{gathered}
$$

Nuevamente, dado que el tick del Contrato "C" es de 0,05 ctvos/lb y que lo que se tiene es una desigualdad, entonces se aproxima hacia arriba tomando el Precio Futuro:

$$
F_{B_{i, j+1}, T_{1}}=\left\langle\text { Redondear }\left\{\frac{\left[F_{h_{i, j}, T_{1}}-\left(\frac{M_{M}-M_{I}}{m}\right) \gamma\right]}{\gamma}\right\}\right\rangle * \gamma
$$

Una vez se cuenta, para la trayectoria $i=1$, con los sucesivos puntos $\left\{F_{h_{i, 1}, T}, F_{h_{i, 2}, T}\right.$, $\left.F_{h_{i, 3}, T}, \ldots\right\}$ y momentos en el tiempo $\left\{h_{i, 1}, h_{i, 2}, h_{i, 3}, \ldots\right\}$ en los que se pueden producir los llamados al margen, se procede a calcular el mismo en cada caso. Se tiene así el siguiente paso (Mejía, 2014): 
Esquema I - Paso 4. Una vez se cuenta, para la trayectoria $i=1$, con los $F_{h_{i, j} T}$ Precios Futuros efectivos que en los sucesivos momentos $h_{i, j}$ generan llamados almargen, se prosigue a calcular los mismos en cada uno de esos tiempos:

$$
M C_{i, j}=-\left(\frac{F_{h_{i, j}, T}-F_{h_{i, j+1}, T}}{\gamma}\right) m x
$$

\section{Donde}

MCi,j es el llamado al margen j para la trayectoria $i$

\section{Alternativamente}

$$
M C_{i, j} \text { es el llamado al margen en el momento } t=h_{i, j}
$$

Con base en lo anterior, el lector notará que se termina teniendo un $(i=1)$ Flujo de Caja, con diversos egresos (y otros en cero) para los distintos momentos del tiempo $t$, tales que $p \geq t \geq f$, denotado como $F C_{1}$. Un ejemplo de este flujo se presenta en el gráfico 5 .

Gráfico 5. Posible dinámica de los llamados al margen en el escenario de backtesting

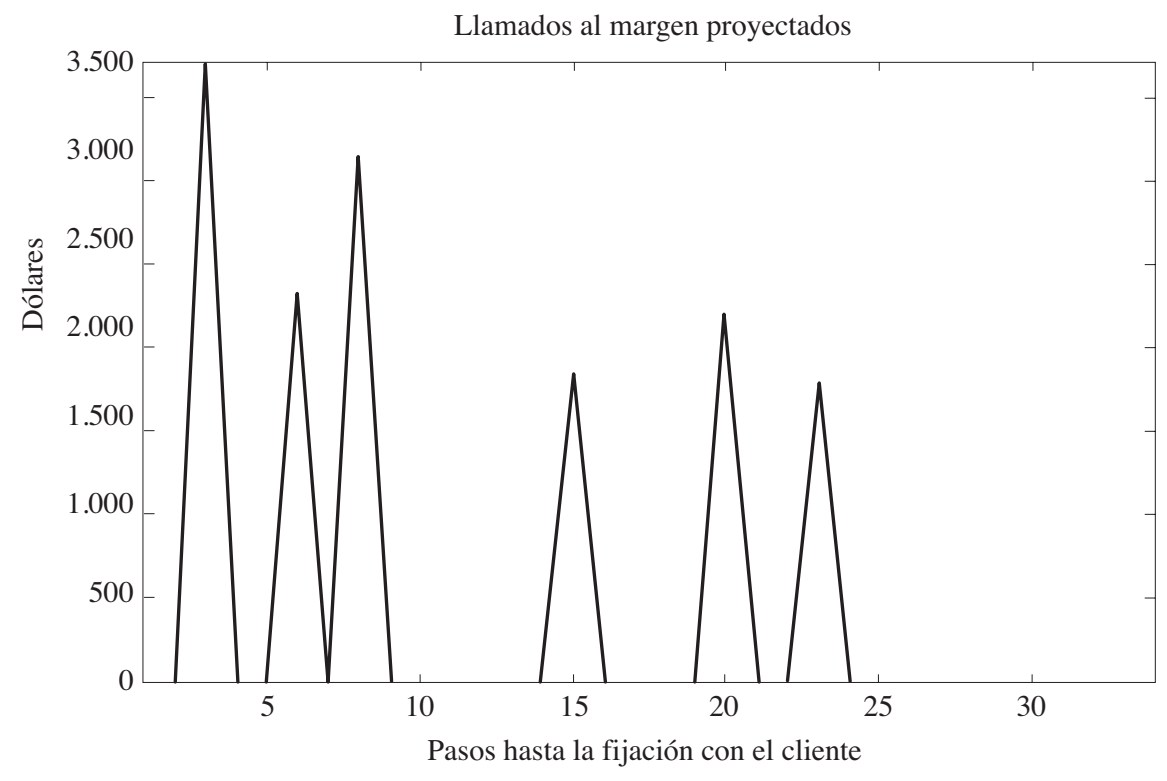


Llamado al margen para una trayectoria del Precio Futuro, proyectado desde el 19 de mayo hasta el 20 de septiembre, dado un Precio Futuro $F(0, T)=133,80$. Proyección bajo el modelo de Gibson y Schwartz (1990), el segundo de Schwartz (1997) o de Brennan y Schwartz (1985) con Media diaria de $\mu=0,3804 \%$, Desviación Estándar diaria de $\sigma=2,2563 \%$ y Tasa Libre de Riesgo $r_{f}=0,0019 \%$ (anexo 5) (Mejía, 2014).

Finalmente, y dado que hasta el momento se cuenta con una sola trayectoria y un solo Flujo de Caja, los pasos I, II, III y IV se realizan veces más (Mejía, 2014).

Esquema I - Paso 5. Una vez se cuenta con el Flujo de Caja $i=1$, se procede a obtener los otros $i+1$ flujos repitiendo los pasos 1,2,3 y 4 .

Con base en lo anterior, el lector notará que ahora se tiene $i$ Flujos de Caja $F C_{i}$. Se procede entonces a sacar el valor esperado, por momento del tiempo $t$, tal que $p \geq t \geq f$, (corte transversal) de cada unos de estos flujos. Posteriormente, se procede a descontar cada uno de estos valores esperados a la Tasa Libre de Riesgo y, finalmente, se suman para obtener el monto del fondo de prevención (Mejía, 2014) ${ }^{49}$ :

Esquema I - Paso 6. Una vez se cuenta con los $i$ Flujos de Caja $F C_{i}$, se procede a sacar el valor esperado de todas las trayectorias por momento del tiempo $t$, tales que $p \geq t \geq f$ (corte transversal). Posteriormente, cada uno de estos valores esperados ${ }^{50}$ se descuenta a la Tasa Libre de Riesgo ${ }^{51}$ y finalmente se suman.

$$
F P_{p}=\sum_{t=p+1}^{f} E_{t}\left[F C_{i}\right] e^{-r f(t-p)}
$$

Donde:

$F P_{p}$ : monto del fondo preventivo en el tiempo $t=p$

49 La adopción de este último paso puede resultar para el lector controversial en la medida que obedece simplemente a que por ser este trabajo una primera aproximación a la administración del riesgo temporal de liquidez asociado a los llamados al margen, se decidió trabajar con el estadístico más elemental (el valor esperado). Sin embargo, no se descarta la posibilidad de optar por otras alternativas y se invita al lector, por el contrario, a desarrollar otros métodos.

50 El valor esperado que se toma se hace bajo la Medida de Probabilidad $Q$, ya que la proyección del Precio Futuro se efectuó con el modelo de Riesgo neutralidad (Schwartz, 1997).

51 El lector notará que, si bien no se hace explícito, este descuento se hace a la Tasa Libre de Riesgo, en cuanto todo el modelo de proyección se trabajó bajo la Medida de Probabilidad $Q$.

PP. $173-231 \cdot N .^{\circ} 9 / 2015$ 
Finalmente, si se aplican los seis pasos al escenario de backtesting, se obtiene un monto para el fondo de US\$16.503. Si estos recursos se depositan directamente en la cuenta de margen se presentaría la siguiente situación (tabla 3):

Tabla 3. Esquema I con recursos en la cuenta de margen

\begin{tabular}{|c|c|c|c|c|c|c|}
\hline Días & $\begin{array}{l}\text { Precio } \\
\text { Futuro }\end{array}$ & $\begin{array}{c}\text { Ganancia } \\
\text { (pérdida) } \\
\text { Diaria }\end{array}$ & $\begin{array}{l}\text { Ganancia } \\
\text { (pérdida) } \\
\text { Acumulada }\end{array}$ & $\begin{array}{l}\text { Balance } \\
\text { Cuenta } \\
\text { Margen }\end{array}$ & $\begin{array}{c}\text { Llamado al } \\
\text { margen }\end{array}$ & $\begin{array}{c}\text { Balance } \\
\text { Cuenta } \\
\text { Margen } \\
\text { Final }\end{array}$ \\
\hline $19 / 05 / 2010$ & 133,80 & & & 22.733 & & 22.733 \\
\hline $20 / 05 / 2010$ & 132,95 & 638 & 638 & 23.371 & - & 23.371 \\
\hline $21 / 05 / 2010$ & 133,70 & (563) & 75 & 22.808 & - & 22.808 \\
\hline $24 / 05 / 2010$ & 133,65 & 37 & 113 & 22.846 & - & 22.846 \\
\hline $25 / 05 / 2010$ & 134,25 & $(450)$ & (337) & 22.396 & - & 22.396 \\
\hline $26 / 05 / 2010$ & 135,25 & $(750)$ & $(1.087)$ & 21.646 & - & 21.646 \\
\hline $27 / 05 / 2010$ & 136,30 & $(788)$ & $(1.875)$ & 20.858 & - & 20.858 \\
\hline $28 / 05 / 2010$ & 135,80 & 375 & $(1.500)$ & 21.233 & - & 21.233 \\
\hline 01/06/2010 & 138,05 & (1.688) & (3.188) & 19.546 & - & 19.546 \\
\hline 02/06/2010 & 136,90 & 863 & (2.325) & 20.408 & - & 20.408 \\
\hline $03 / 06 / 2010$ & 137,05 & (113) & (2.438) & 20.296 & - & 20.296 \\
\hline 04/06/2010 & 135,30 & 1.313 & (1.125) & 21.608 & - & 21.608 \\
\hline $07 / 06 / 2010$ & 134,60 & 525 & $(600)$ & 22.133 & - & 22.133 \\
\hline 08/06/2010 & 135,10 & (375) & (975) & 21.758 & - & 21.758 \\
\hline 09/06/2010 & 136,05 & (713) & (1.688) & 21.046 & - & 21.046 \\
\hline 10/06/2010 & 138,55 & (1.875) & (3.563) & 19.171 & - & 19.171 \\
\hline $11 / 06 / 2010$ & 146,10 & (5.662) & (9.225) & 13.508 & - & 13.508 \\
\hline $14 / 06 / 2010$ & 151,60 & (4.125) & $(13.350)$ & 9.383 & - & 9.383 \\
\hline $15 / 06 / 2010$ & 159,95 & $(6.263)$ & (19.613) & 3.121 & 3.109 & 6.230 \\
\hline $16 / 06 / 2010$ & 159,60 & 262 & $(19.350)$ & 6.493 & - & 6.493 \\
\hline $17 / 06 / 2010$ & 157,80 & 1.350 & $(18.000)$ & 7.842 & - & 7.842 \\
\hline $18 / 06 / 2010$ & 162,10 & (3.225) & $(21.225)$ & 4.618 & 1.613 & 6.230 \\
\hline $21 / 06 / 2010$ & 160,80 & 975 & $(20.250)$ & 7.205 & - & 7.205 \\
\hline $22 / 06 / 2010$ & 160,75 & 38 & $(20.213)$ & 7.243 & - & 7.243 \\
\hline $23 / 06 / 2010$ & 160,50 & 188 & (20.025) & 7.430 & - & 7.430 \\
\hline 24/06/2010 & 168,75 & (6.188) & $(26.213)$ & 1.243 & 4.988 & 6.230 \\
\hline $25 / 06 / 2010$ & 168,90 & (113) & $(26.325)$ & 6.118 & - & 6.118 \\
\hline $28 / 06 / 2010$ & 168,10 & 600 & $(25.725)$ & 6.718 & - & 6.718 \\
\hline 29/06/2010 & 163,15 & 3.712 & (22.013) & 10.430 & - & 10.430 \\
\hline $30 / 06 / 2010$ & 165,85 & (2.025) & $(24.038)$ & 8.405 & - & 8.405 \\
\hline $01 / 07 / 2010$ & 168,25 & $(1.800)$ & $(25.838)$ & 6.605 & - & 6.605 \\
\hline $02 / 07 / 2010$ & 164,30 & 2.962 & $(22.875)$ & 9.567 & - & 9.567 \\
\hline $06 / 07 / 2010$ & 157,65 & 4.988 & $(17.888)$ & 14.555 & - & 14.555 \\
\hline $07 / 07 / 2010$ & 163,05 & $(4.050)$ & $(21.938)$ & 10.505 & - & 10.505 \\
\hline
\end{tabular}

Tomado de Mejía (2014). 
$\mathrm{Si}$, por el contrario, los recursos se mantienen en una cuenta y se utilizan ante los sucesivos llamados al margen, se presentaría la siguiente situación (tabla 4):

Tabla 4. Esquema I con recursos en otra cuenta

Cobertura 1

\begin{tabular}{|c|c|c|c|c|c|c|c|c|}
\hline Días & $\begin{array}{l}\text { Precio } \\
\text { Futuro }\end{array}$ & $\begin{array}{c}\text { Ganancia } \\
\text { (pérdida) } \\
\text { Diaria }\end{array}$ & $\begin{array}{c}\text { Ganancia } \\
\text { (pérdida) } \\
\text { Acumulada }\end{array}$ & $\begin{array}{l}\text { Balance } \\
\text { Cuenta } \\
\text { Margen }\end{array}$ & $\begin{array}{c}\text { Llamado al } \\
\text { margen }\end{array}$ & $\begin{array}{c}\text { Balance } \\
\text { Cuenta } \\
\text { Margen } \\
\text { Final }\end{array}$ & Fondo & $\begin{array}{c}\text { Fondo } \\
\text { ajustado }\end{array}$ \\
\hline $19 / 05 / 2010$ & 133,80 & & & 6.230 & & 6.230 & 16.503 & 16.503 \\
\hline $20 / 05 / 2010$ & 132,95 & 638 & 638 & 6.868 & - & 6.868 & 16.503 & 16.503 \\
\hline $21 / 05 / 2010$ & 133,70 & (563) & 75 & 6.305 & - & 6.305 & 16.503 & 16.503 \\
\hline $24 / 05 / 2010$ & 133,65 & 37 & 113 & 6.343 & - & 6.343 & 16.503 & 16.503 \\
\hline $25 / 05 / 2010$ & 134,25 & $(450)$ & (337) & 5.893 & - & 5.893 & 16.503 & 16.503 \\
\hline $26 / 05 / 2010$ & 135,25 & $(750)$ & $(1.087)$ & 5.143 & - & 5.143 & 16.503 & 16.503 \\
\hline $27 / 05 / 2010$ & 136,30 & $(788)$ & (1.875) & 4.355 & 1.875 & 6.230 & 16.503 & 14.628 \\
\hline 28/05/2010 & 135,80 & 375 & (1.500) & 6.605 & - & 6.605 & 14.628 & 14.628 \\
\hline $01 / 06 / 2010$ & 138,05 & (1.688) & (3.188) & 4.918 & 1.313 & 6.230 & 14.628 & 13.316 \\
\hline $02 / 06 / 2010$ & 136,90 & 863 & (2.325) & 7.093 & - & 7.093 & 13.316 & 13.316 \\
\hline 03/06/2010 & 137,05 & (113) & (2.438) & 6.980 & - & 6.980 & 13.316 & 13.316 \\
\hline $04 / 06 / 2010$ & 135,30 & 1.313 & (1.125) & 8.293 & - & 8.293 & 13.316 & 13.316 \\
\hline 07/06/2010 & 134,60 & 525 & (600) & 8.818 & - & 8.818 & 13.316 & 13.316 \\
\hline $08 / 06 / 2010$ & 135,10 & (375) & (975) & 8.443 & - & 8.443 & 13.316 & 13.316 \\
\hline 09/06/2010 & 136,05 & (713) & (1.688) & 7.730 & - & 7.730 & 13.316 & 13.316 \\
\hline $10 / 06 / 2010$ & 138,55 & $(1.875)$ & (3.563) & 5.855 & - & 5.855 & 13.316 & 13.316 \\
\hline $11 / 06 / 2010$ & 146,10 & $(5.662)$ & (9.225) & 193 & 6.037 & 6.230 & 13.316 & 7.278 \\
\hline $14 / 06 / 2010$ & 151,60 & (4.125) & (13.350) & 2.105 & 4.125 & 6.230 & 7.278 & 3.153 \\
\hline $15 / 06 / 2010$ & 159,95 & $(6.263)$ & (19.613) & (32) & 6.263 & 6.230 & 3.153 & (3.109) \\
\hline $16 / 06 / 2010$ & 159,60 & 262 & (19.350) & 6.493 & - & 6.493 & (3.109) & (3.109) \\
\hline $17 / 06 / 2010$ & 157,80 & 1.350 & $(18.000)$ & 7.842 & - & 7.842 & (3.109) & (3.109) \\
\hline $18 / 06 / 2010$ & 162,10 & (3.225) & (21.225) & 4.618 & 1.613 & 6.230 & (3.109) & (4.722) \\
\hline $21 / 06 / 2010$ & 160,80 & 975 & (20.250) & 7.205 & - & 7.205 & $(4.722)$ & (4.722) \\
\hline $22 / 06 / 2010$ & 160,75 & 38 & (20.213) & 7.243 & - & 7.243 & (4.722) & (4.722) \\
\hline $23 / 06 / 2010$ & 160,50 & 188 & $(20.025)$ & 7.430 & - & 7.430 & $(4.722)$ & (4.722) \\
\hline $24 / 06 / 2010$ & 168,75 & (6.188) & (26.213) & 1.243 & 4.988 & 6.230 & (4.722) & (9.709) \\
\hline 25/06/2010 & 168,90 & (113) & (26.325) & 6.118 & - & 6.118 & (9.709) & (9.709) \\
\hline $28 / 06 / 2010$ & 168,10 & 600 & $(25.725)$ & 6.718 & - & 6.718 & $(9.709)$ & (9.709) \\
\hline 29/06/2010 & 163,15 & 3.712 & (22.013) & 10.430 & - & 10.430 & (9.709) & (9.709) \\
\hline $30 / 06 / 2010$ & 165,85 & $(2.025)$ & (24.038) & 8.405 & - & 8.405 & (9.709) & (9.709) \\
\hline $01 / 07 / 2010$ & 168,25 & $(1.800)$ & (25.838) & 6.605 & - & 6.605 & (9.709) & (9.709) \\
\hline $02 / 07 / 2010$ & 164,30 & 2.962 & (22.875) & 9.567 & - & 9.567 & (9.709) & (9.709) \\
\hline 06/07/2010 & 157,65 & 4.988 & (17.888) & 14.555 & - & 14.555 & (9.709) & (9.709) \\
\hline $07 / 07 / 2010$ & 163,05 & $(4.050)$ & (21.938) & 10.505 & - & 10.505 & $(9.709)$ & (9.709) \\
\hline
\end{tabular}

Tomado de Mejía (2014). 
Como se aprecia en la tabla 4, el exportador pasaría de buscar US\$26.213 a tan solo US\$ 9.709 para garantizar que el esquema de cobertura de precio se mantenga y se obtenga el diferencial neto de $15 \mathrm{ctvos} / \mathrm{lb}$. Asimismo, es importante resaltar que independientemente de si el monto se deposita en la cuenta de margen o si se mantiene en una cuenta aparte y se recurre a ella en el momento de presentarse los llamados al margen ${ }^{52}$, estos últimos se presentarían en los mismos momentos del tiempo y por los mismos montos (Mejía, 2014).

\subsection{Esquema condicionado en el que se va recalculando el monto del fondo preventivo a medida que pasa el tiempo}

Como se aprecia en las tablas 3 y 4 , el esquema anterior mitiga una porción importante del riesgo temporal de liquidez asociado a los llamados al margen, pero no en su totalidad. Una de las razones por las que se presenta lo anterior, es debido a la existencia de clusters de volatilidad que no logran capturar el modelo de proyección al asumir que la desviación estándar instantánea es constante ${ }^{53}$. Lo anterior supone que para optimizar el esquema de administración, existen dos soluciones: i) incorporar el carácter determinístico o estocástico de la desviación estándar en el modelo planteado anteriormente ${ }^{54} \mathrm{o}$ ii) manejar un esquema condicionado en el que el monto del fondo se va ajustando a medida que se revela nueva información de mercado (Mejía, 2014).

La diferencia entre el esquema I y este segundo esquema consiste en que los seis pasos se repiten sucesivamente para cada $t=1$, tal que $p<l<f$, en la medida que se cuenta con nueva información de los nuevos cierres que se generan, ajustando

52 Esto se debe a que en el presente trabajo no se contempla que los recursos en una u otra cuenta renten algo o que exista diferencial de tasas a las que rentan los recursos en una cuenta de margen frente a otra cuenta cualquiera. Al igual que muchos otros puntos, se invita al lector a profundizar en estos temas en futuros trabajos.

53 En realidad, una razón de mayor peso por la que se puede presentar esto se debe al último paso que se propone para calcular el monto del fondo preventivo asociado con el valor esperado. Una posible forma de resolver lo anterior se presentará en las conclusiones.

54 Lo anterior constituye un incentivo para aplicar el esquema estático modificando el supuesto utilizado en la presente tesis respecto a la desviación estándar instantánea, dejando que esta dependa del tiempo, o sea, del carácter estocástico. Se advierte que uno de los problemas que se puede llegar a encontrar con este enfoque es el asociado con la completitud del mercado, en cuanto se tendrían ahora dos fuentes de incertidumbre (la relativa al Precio Spot Estándar y la de la desviación estándar instantánea) con un solo instrumento de cobertura (Helfrich, 2009). 
por tanto el monto del fondo sucesivamente hasta que $t=f$. Lo anterior implica que por momentos se tengan excedentes o faltantes a los cuales se les debe dar el siguiente trato (Mejía, 2014):

- Los excedentes se mantienen en la cuenta para futuras contingencias.

- Los faltantes se deben suplir hasta obtener el monto del fondo requerido.

Bajo este escenario, el Precio Futuro del Contrato "C" con vencimiento a septiembre, que se utiliza de referencia el 19 de mayo de 2010 para la compra del cPs es de 133,80 Ctvs / lb. Al igual que para el esquema estático, con este se obtiene el primer monto del fondo de US\$16.503. Sin embargo, una vez transcurre el primer día y el contrato futuro cierra el 20 de mayo de 2013 en 132,95 ctvos/lb., se ajusta otra vez el modelo con los nuevos parámetros (anexo 5) y se obtiene un nuevo monto del fondo de US\$ 15.744. De esta forma, se tiene un excedente US\$759, por lo que no se realizan aumentos al fondo (Mejía, 2014). El proceso continúa como se muestra en la tabla 5.

Tabla 5. Esquema II

\begin{tabular}{|c|c|c|c|c|c|c|c|c|c|c|c|c|}
\hline Días & $\begin{array}{l}\text { Precio } \\
\text { Futuro }\end{array}$ & $\begin{array}{c}\text { Ganancia } \\
\text { (pérdida) } \\
\text { Diaria }\end{array}$ & $\begin{array}{c}\text { Ganancia } \\
\text { (pérdida) } \\
\text { Acumulada }\end{array}$ & $\begin{array}{l}\text { Balance } \\
\text { Cuenta } \\
\text { Margen }\end{array}$ & $\begin{array}{l}\text { Ulamado al } \\
\text { margen }\end{array}$ & $\begin{array}{c}\text { Balance } \\
\text { Cuenta } \\
\text { Margen } \\
\text { Final }\end{array}$ & $\begin{array}{l}\text { Fondo } \\
\text { Inicial }\end{array}$ & $\begin{array}{c}\text { Fondo } \\
\text { ajustado }\end{array}$ & $\begin{array}{c}\text { Aumentos } \\
\text { fondo }\end{array}$ & $\begin{array}{c}\text { Fondo } \\
\text { final }\end{array}$ & $\begin{array}{l}\text { Fondo } \\
\text { teórico }\end{array}$ & $\%$ \\
\hline $19 / 05 / 2010$ & 133,80 & & & 6.230 & & 6.230 & - & - & 16.503 & 16.503 & 16.503 & \\
\hline $20 / 05 / 2010$ & 132,95 & 638 & 638 & 6.868 & - & 6.868 & 16.503 & 16.503 & - & 16.503 & 15.744 & \\
\hline $21 / 05 / 2010$ & 133,70 & (563) & 75 & 6.305 & - & 6.305 & 16.503 & 16.503 & - & 16.503 & 16.143 & \\
\hline $24 / 05 / 2010$ & 133,65 & 37 & 113 & 6.343 & - & 6.343 & 16.503 & 16.503 & - & 16.503 & 15.652 & \\
\hline $25 / 05 / 2010$ & 134,25 & (450) & (337) & 5.893 & - & 5.893 & 16.503 & 16.503 & - & 16.503 & 15.821 & \\
\hline $26 / 05 / 2010$ & 135,25 & (750) & $(1.087)$ & 5.143 & - & 5.143 & 16.503 & 16.503 & - & 16.503 & 16.092 & \\
\hline $27 / 05 / 2010$ & 136,30 & (788) & (1.875) & 4.355 & 1.875 & 6.230 & 16.503 & 14.628 & 29 & 14.657 & 14.657 & $2 \%$ \\
\hline $28 / 05 / 2010$ & 135,80 & 375 & $(1.500)$ & 6.605 & - & 6.605 & 14.657 & 14.657 & - & 14.657 & 14.054 & \\
\hline $01 / 06 / 2010$ & 138,05 & (1.688) & (3.188) & 4.918 & 1.313 & 6.230 & 14.657 & 13.345 & 498 & 13.842 & 13.842 & $38 \%$ \\
\hline $02 / 06 / 2010$ & 136,90 & 863 & (2.325) & 7.093 & - & 7.093 & 13.842 & 13.842 & - & 13.842 & 12.991 & \\
\hline $03 / 06 / 2010$ & 137,05 & (113) & (2.438) & 6.980 & - & 6.980 & 13.842 & 13.842 & - & 13.842 & 12.720 & \\
\hline $04 / 06 / 2010$ & 135,30 & 1.313 & (1.125) & 8.293 & - & 8.293 & 13.842 & 13.842 & - & 13.842 & 11.305 & \\
\hline $07 / 06 / 2010$ & 134,60 & 525 & (600) & 8.818 & - & 8.818 & 13.842 & 13.842 & - & 13.842 & 10.426 & \\
\hline $08 / 06 / 2010$ & 135,10 & (375) & (975) & 8.443 & - & 8.443 & 13.842 & 13.842 & - & 13.842 & 10.516 & \\
\hline $09 / 06 / 2010$ & 136,05 & (713) & (1.688) & 7.730 & - & 7.730 & 13.842 & 13.842 & - & 13.842 & 10.819 & \\
\hline $10 / 06 / 2010$ & 138,55 & $(1.875)$ & (3.563) & 5.855 & - & 5.855 & 13.842 & 13.842 & - & 13.842 & 11.665 & \\
\hline $11 / 06 / 2010$ & 146,10 & (5.662) & $(9.225)$ & 193 & 6.037 & 6.230 & 13.842 & 7.805 & 2.472 & 10.277 & 10.277 & $41 \%$ \\
\hline $14 / 06 / 2010$ & 151,60 & $(4.125)$ & (13.350) & 2.105 & 4.125 & 6.230 & 10.277 & 6.152 & 3.742 & 9.894 & 9.894 & $91 \%$ \\
\hline $15 / 06 / 2010$ & 159,95 & $(6.263)$ & (19.613) & (32) & 6.263 & 6.230 & 9.894 & 3.631 & 4.880 & 8.511 & 8.511 & $78 \%$ \\
\hline $16 / 06 / 2010$ & 159,60 & 262 & (19.350) & 6.493 & - & 6.493 & 8.511 & 8.511 & - & 8.511 & 7.947 & \\
\hline $17 / 06 / 2010$ & 157,80 & 1.350 & $(18.000)$ & 7.842 & - & 7.842 & 8.511 & 8.511 & - & 8.511 & 6.506 & \\
\hline $18 / 06 / 2010$ & 162,10 & (3.225) & (21.225) & 4.618 & 1.613 & 6.230 & 8.511 & 6.898 & 526 & 7.424 & 7.424 & $33 \%$ \\
\hline $21 / 06 / 2010$ & 160,80 & 975 & $(20.250)$ & 7.205 & - & 7.205 & 7.424 & 7.424 & - & 7.424 & 6.218 & \\
\hline $22 / 06 / 2010$ & 160,75 & 38 & $(20.213)$ & 7.243 & - & 7.243 & 7.424 & 7.424 & - & 7.424 & 5.619 & \\
\hline $23 / 06 / 2010$ & 160,50 & 188 & $(20.025)$ & 7.430 & - & 7.430 & 7.424 & 7.424 & - & 7.424 & 4.887 & \\
\hline $24 / 06 / 2010$ & 168,75 & (6.188) & (26.213) & 1.243 & 4.988 & 6.230 & 7.424 & 2.436 & 2.643 & 5.079 & 5.079 & $53 \%$ \\
\hline $25 / 06 / 2010$ & 168,90 & (113) & (26.325) & 6.118 & - & 6.118 & 5.079 & 5.079 & - & 5.079 & 4.709 & \\
\hline $28 / 06 / 2010$ & 168,10 & 600 & (25.725) & 6.718 & - & 6.718 & 5.079 & 5.079 & - & 5.079 & 3.737 & \\
\hline $29 / 06 / 2010$ & 163,15 & 3.712 & $(22.013)$ & 10.430 & - & 10.430 & 5.079 & 5.079 & - & 5.079 & 1.222 & \\
\hline $30 / 06 / 2010$ & 165,85 & $(2.025)$ & (24.038) & 8.405 & - & 8.405 & 5.079 & 5.079 & - & 5.079 & 1.565 & \\
\hline $01 / 07 / 2010$ & 168,25 & $(1.800)$ & (25.838) & 6.605 & - & 6.605 & 5.079 & 5.079 & - & 5.079 & 1.819 & \\
\hline $02 / 07 / 2010$ & 164,30 & 2.962 & (22.875) & 9.567 & - & 9.567 & 5.079 & 5.079 & - & 5.079 & 166 & \\
\hline $06 / 07 / 2010$ & 157,65 & 4.988 & (17.888) & 14.555 & - & 14.555 & 5.079 & 5.079 & - & 5.079 & - & \\
\hline $07 / 07 / 2010$ & 163,05 & $(4.050)$ & (21.938) & 10.505 & - & 10.505 & 5.079 & 5.079 & - & 5.079 & - & \\
\hline
\end{tabular}

Tomado de Mejía (2014).

PP. $173-231$ • N. ${ }^{\circ} 9 / 2015$ 
Como se aprecia en la tabla 5, este esquema de administración tiene varias ventajas (Mejía, 2014):

- En los momentos en los que se presentan llamados al margen, el exportador contaría con el fondo para cumplir con el mismo y así evitar el riesgo temporal de liquidez asociado.

- Los momentos en los que se requiere incrementar el monto del fondo coinciden en todos los casos con situaciones en las que se produciría en esa fecha un llamado al margen. Sin embargo, se presentan dos ventajas respecto al llamado al margen original: i) los requerimientos siempre son inferiores y ii) en caso de no poder cumplir con el mismo, se puede responder posteriormente sin que por ellos se deba cerrar la posición en los contratos futuros ${ }^{55}$.

\section{Conclusiones, soluciones parciales y extensiones}

El objetivo del presente artículo fue mostrar brevemente los resultados de la tesis de grado "Administración del riesgo temporal de liquidez asociado a los llamados al margen dentro del negocio de comercialización del café verde en Colombia", en la cual se busco una primera aproximación de esquemas que permitieran administrar el riesgo temporal de liquidez que conllevan los llamados al margen. En dicho trabajo, se concluyó que si se optara por estructurar un fondo al momento de tomar la posición corta en el Contrato " $\mathrm{C}$ " y mantenerlo hasta que se fije el precio con el cliente final (esquema I), se podrían cumplir los requerimientos hasta cierto punto. Por otro lado, con el segundo esquema en el que se va actualizando el monto en cada momento del tiempo, dada la nueva información de mercado, se concluyó que en prácticamente todos los momentos del tiempo que se presentan llamados al margen, se debe aumentar también el monto del fondo. Sin embargo, el valor que se va a colocar resulta ser mucho menor al requerimiento por parte del broker y, adicionalmente, no debe efectuarse inmediatamente sino que puede esperar algunos días (Mejía, 2014).

\footnotetext{
55 Para el caso puntual, al cierre de los días 11,14 y 15 se producen importantes llamados al margen por US\$16.425. Si alguno de ellos no se cumple, se perdería la posición en los contratos futuros. Por el contario, con este esquema el requerimiento para cumplir con los aumentos sería de US\$11.094. Sin embargo, si no se pudiera cumplir con algunos de los tres requerimientos de inmediato no se desarma la estrategia de cobertura.
} 
Sin embargo, a pesar de estos beneficios, lo cierto es que aún se deben depositar recursos adicionales que muestran que el fondo preventivo aún no es sostenible por sí mismo para atender todos los llamados al margen. Lo anterior lleva a pensar que quizá el problema no radique tanto en el manejo de la desviación estándar instantánea como constante (aunque no por ello deja de ser importante), sino por alguna otra razón. Al revisar detenidamente, resulta evidente que en el paso 4, una vez se cuenta con una serie de trayectorias de llamados al margen, se tomó una decisión que en primera instancia no parecía requerir de mucho análisis, pero que a la luz de la evidencia, puede resultar fundamental: el uso del estadístico de primer momento más elemental, es decir, el valor esperado para el cálculo final del monto del fondo preventivo. Por tanto, se puede concluir que la constitución de un fondo preventivo para administrar el riesgo temporal de liquidez asociado a los llamados al margen dentro del negocio de comercialización de café verde en Colombia no podría estructurarse a partir del valor esperado (Mejía, 2014).

Ahora bien, el emplear el valor esperado radicaba en creer que este era un estimador no sesgado, hecho que se produce cuando se asume que la distribución de probabilidad de la serie de llamados al margen es normal. Por tanto, una posible solución sería la de encontrar la distribución real y para ello se puede dividir la serie en dos partes, inspirándonos en la dualidad vista entre el Precio Futuro Barrera y el Precio Futuro Efectivo (Mejía, 2014):

- Como se pudo apreciar anteriormente, la primera parte del problema consistía en determinar los sucesivos Precios Futuros Barrera y ver en qué puntos el Precio Futuro proyectado los tocaba a lo largo del tiempo desde $p>t>f$.

- Por otro lado, la segunda parte del problema consistía en mirar en cada uno de esos puntos o momentos en el tiempo los Precios Futuros Efectivos y calcular en cada uno el respectivo llamado al margen a lo largo del tiempo desde $p>t>f$.

Ahora bien, con la ayuda de la ecuación 12, y siguiendo todos los pasos hasta el número 6, se aprecia que, finalmente, se tienen una serie de trayectorias de llamados al margen. A partir de ahí, y con base en la división del problema, se puede proponer lo siguiente (Mejía, 2014):

- Si se le asocia a cada uno de los llamados al margen un uno (1) y a los ceros en los que no se produce un cero $(0)$, se termina así con una serie de vectores que PP. $173-231 \cdot N .^{\circ} 9 / 2015$ 
indican en qué puntos se tocan las sucesivas barreras. De esta forma, se puede buscar la distribución que mejor se ajusta a este conjunto de datos. Con ayuda de la herramienta Crystall Ball, y para el caso particular del backtesting, se obtiene una distribución binomial negativa con probabilidad 32,814\% y forma $2^{56}$, como se aprecia en el gráfico 6.

Gráfico 6. Ajuste de la distribución para el problema de las barreras.

Distribución binomial inversa

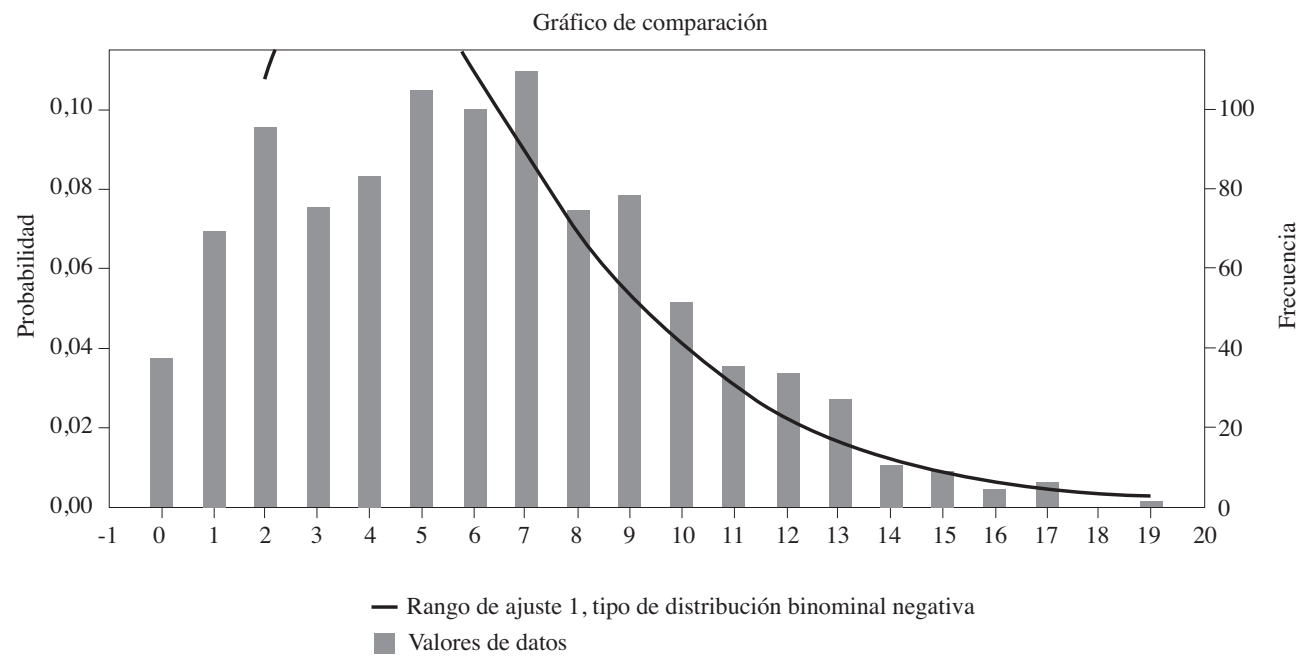

Tomado de Mejía (2014).

- Por otro lado, la misma base de datos contiene en cada uno de esos puntos el monto del posible llamado al margen. De esta forma, se puede buscar la distribución que mejor se ajusta a este conjunto de datos. Con ayuda de la herramienta Crystall Ball, y para el caso particular del backtesting, se obtiene la siguiente distribución logística con media 4.498,93 y escala 1.039,3157 como se aprecia en el gráfico 7.

56 La herramienta fitting de Crystall Ball permite identificar la posible distribución que tiene una serie de datos. En ese sentido, dada la proyección de ceros y unos, se construyó una nueva serie, de la forma vector columna, en la que en cada celda se totalizaban los unos (1) y los ceros (0) (indicando, por tanto, el número de llamados al margen producidos por cada trayectoria proyectada) y se hizo uso de la herramienta fitting para identificar la distribución que puede llegar a tener dicha serie.

57 La herramienta fitting de Crystall Ball permite identificar la posible distribución que tiene una serie de datos. En ese sentido, dada la proyección de llamados al margen, se construyó una 
Gráfico 7. Ajuste de la distribución para el problema de los llamados al margen. Distribución logística

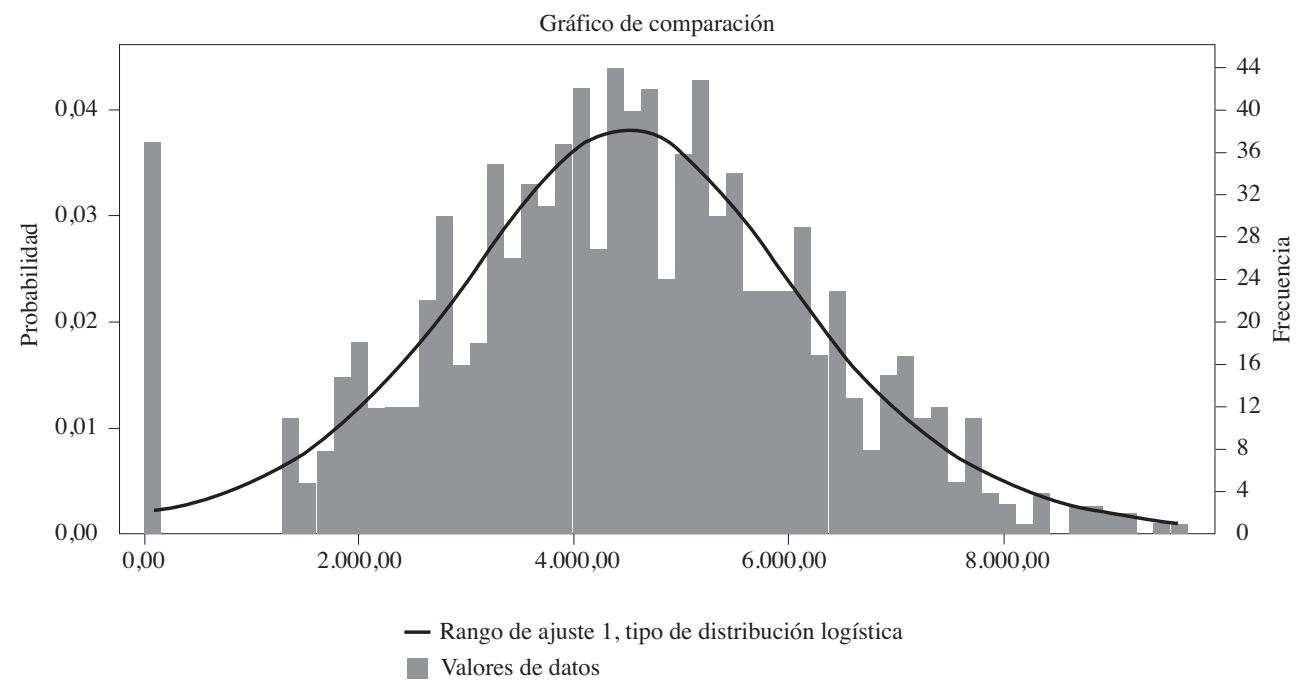

Tomado de Mejía (2014).

- Tan pronto se tienen ambas distribuciones, se puede llevar a cabo un proceso matemático conocido como convolución para obtener una tercera distribución que precisamente indique, dado un nivel de confianza, el monto del fondo preventivo. Una vez más, con ayuda de la herramienta Crystall Ball y para el caso particular del backtesting, se obtiene la siguiente distribución con media de US\$27.430 (gráfico 8) ${ }^{58}$ :

nueva serie, de la forma vector columna, en la que en cada celda se incluía el máximo de los llamado al margen (indicando, por tanto, el llamados al margen más alto por cada trayectoria proyectada) y se hizo uso de la herramienta fitting para identificar la distribución que puede llegar a tener dicha serie.

58 De esta manera, pese a que el cálculo puede resultar superficial, se refleja que incorporando otras formas de cálculo del monto, se puede llegar a una cifra muy cercana a la que se requiere para administrar el riesgo temporal de liquidez. 
Gráfico 8. Distribución que deriva del proceso de la convolución

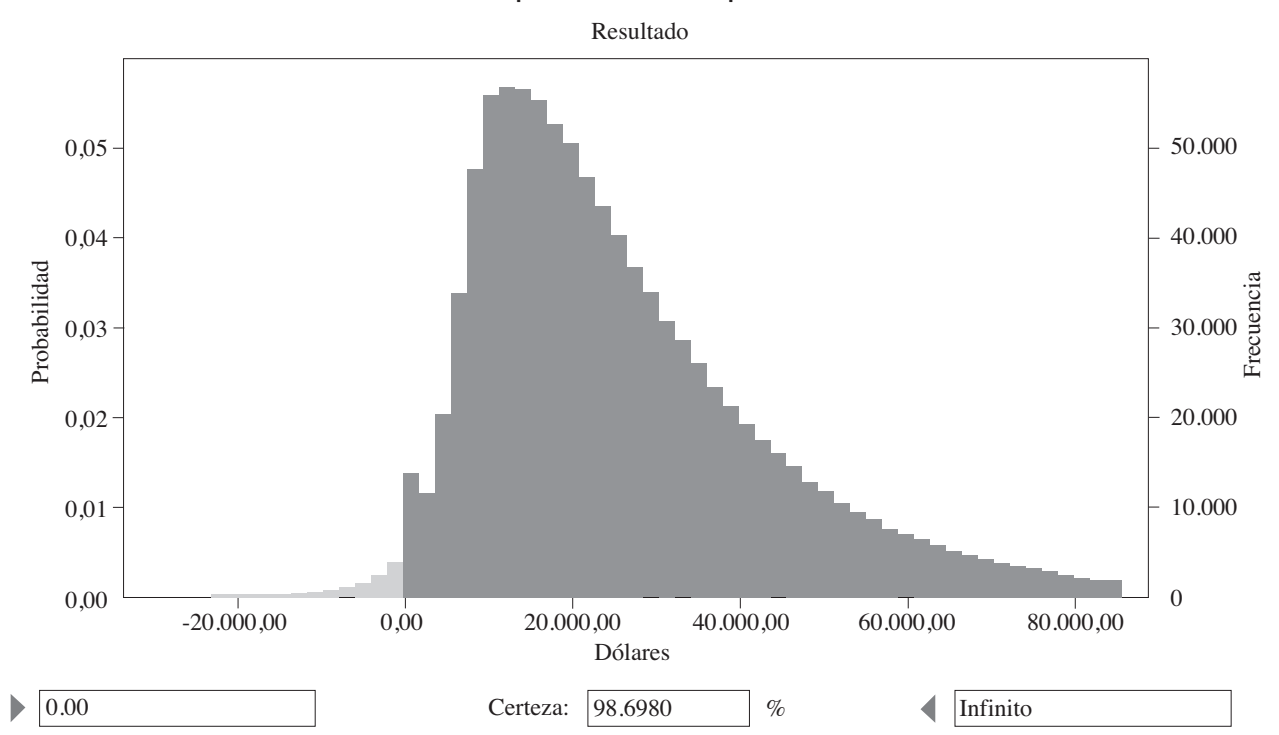

\begin{tabular}{|l|l|r|}
\hline & \multicolumn{1}{|c|}{ Estadística } & \multicolumn{1}{c|}{ Valores de previsión } \\
\hline & Pruebas & 1.000 .000 \\
\hline & Caso base & 0,00 \\
\hline & Media & $27.430,32$ \\
\hline & Mediana & $22.308,46$ \\
\hline & Modo & --- \\
\hline & Desviación estándar & $20.750,44$ \\
\hline & Varianza & 1,82 \\
\hline & Sesgo & 9,11 \\
\hline & Curtosis & 0,7565 \\
\hline & Coeficiente de variación & $-133.003,36$ \\
\hline & Mínimo & $339.320,48$ \\
\hline & Máximo & 20,75 \\
\hline & Error estándar medio & \\
\hline
\end{tabular}

Tomado de Mejía (2014).

Con base en lo anterior, se aprecia que existe un inmenso mundo de posibilidades respecto a la forma en la que puede calcularse el monto del mismo una vez se llega al paso 6 . Ahora bien, se debe señalar que el exportador que opte por algún esquema de cobertura de precio a través del Contrato "C", como el que se presenta en este artículo y que emplee alguno de los esquemas de administración, debe estar dispuesto a sacrificar parte de la rentabilidad que buscaba aislar en primera medida con el esquema de cobertura, ya que mantener recursos en un fondo preventivo 
supone inevitablemente un costo financiero (la Tasa Interna de Oportunidad en caso de recursos propios o la tasa de interés en el caso que provengan de recursos de tercero). Se obliga así al exportador a definir lo anterior como un criterio más dentro de su política de cobertura: si quiero evitar un problema de liquidez debo sacrificar rentabilidad, o, si deseo más rentabilidad, debo exponerme a un mayor riesgo de liquidez (Mejía, 2014).

Finalmente, algunas extensiones adicionales son las siguientes (Mejía, 2014):

- Uno de los elementos que no resulta evidente dentro del apartado 4 al momento de comenzar a construir el fondo de prevención, es que se está trabajando exclusivamente con el downside risk y se está excluyendo el upside risk. Lo anterior resultará evidente para el lector si se fija que los distintos pasos mostrados en el apartado 4 se concentran exclusivamente en los recursos adicionales que se deben depositar en la cuenta de margen cuando el Contrato "C" sube, pero no analiza en absoluto lo que sucede con la misma cuando el Precio Futuro cae y entran recursos a la misma.

- En el presente trabajo se asumió que la Convenience Yield Neta, la Tasa Libre de Riesgo e incluso la desviación instantánea se mantenían constantes a través del tiempo. Sin embargo, si se rompe con estos supuestos (por ejemplo, al contemplar esquemas de cobertura con contratos de mayor plazo o al introducir el hecho de que la cuenta de margen pueda rentar a la Tasa Libre de Riesgo), se debería recurrir a otros modelos más complejos.

- En el capítulo 2 se expuso brevemente uno de los esquemas de cobertura más básicos dentro del mercado internacional de café verde. Se concluyó que a través del mismo se podría mitigar una porción importante del riesgo de precio (la porción estándar), pero aún el exportador quedaba expuesto al riesgo asociado a los diferenciales de compra y venta. En la actualidad, son pocos los trabajos que se han desarrollado acerca de cómo mitigar este riesgo.

- Finalmente, es importante señalar que en el presente trabajo se hizo uso de un commodity (el café verde), de un contrato futuro específico (el Contrato "C") y de un esquema de cobertura de precio básico. Sin embargo, muchos de los aspectos vistos en este trabajo se pueden extender a otros commodities, otros derivados y a otros esquemas de cobertura. 


\section{Referencias}

Bank for International Settlements: Basel Committee on Banking Supervision (2001, 31 de mayo). Operational Risk. Recuperado de: http://www.bis.org/publ/bcbsca07.pdf

Basel Committee on Banking Supervision (2000, septiembre). Principles for the Management of Credit Risk. Recuperado de: http://www.bis.org/publ/bcbs75.pdf

Benhamou, E. (2005, 31 de julio). Black's model (1976). Recuperado de: http://www. ericbenhamou.net/documents/Encyclo/Black's\%20model.pdf

Bloomberg (s.f.).

Bravo Orellana, S. (n. d.). Los parámetros del Capital Asset Pricing Model. Conceptos y Estimación. Recuperado de: http://www.indiceperu.com/lecturas/paper04.pdf

Brennan, M. y Schwartz, E. (1985, abril). Evaluating Natural Resources Investments. Recuperado de: http://legacy.samsi.info/200506/fmse/course-info/fackler/BrennanSchwartz85.pdf

Campbell, J. Y., Andrew, W. L. y MacKinlay, A. C. (1997). The Econometrics of Financial Markets. New Jersey: Princeton University Press.

Cheung, Y. H. y Powell, R. J. (2012). Anybody can do Value at Risk: A Teaching Study using Parametric Computation and Monte Carlo Simulation. Recuperado de: http:// ro.uow.edu.au/cgi/viewcontent.cgi? article $=1400 \&$ context $=$ aabfj

Chicago Mercantile Exchange Group (CME Group) (2012). Self-study guide to Hedging with grain and oilseed Futures and options. Recuperado de: http://www.cmegroup. com/trading/agricultural/files/AC-216_HedgersGuideNewBoilerplate.pdf

Complete Coffee (2008). Green Coffee. Recuperado de: http://www.completecoffee.com/ green_coffee/green_coffee.html

EAFIT (2007). Generalización del negocio de exportación de café verde en Colombia. Recuperado de: http://bdigital.eafit.edu.co/PROYECTO/P382.609861CDV732E/ marcoTeorico.pdf

European Central Bank (2009, febrero). Liquidity (Risk) Concepts: Definitions and Interactions. Recuperado de: http://www.ecb.europa.eu/pub/pdf/scpwps/ecbwp1008.pdf 
Fama, E. F. (1970, mayo). Efficient Capital Markets: A Review of Theory and Empirical Work. Recuperado de: http://efinance.org.cn/cn/fm/Efficient\%20Capital\%20 Markets\%20A\%20Review\%20of\%20Theory\%20and\%20Empirical\%20Work.pdf

Fantacci,L., Marcuzzo, M. C. y Sanfilippo,E. (2010). Speculation in commodities: Keynes "Practical Acquaintance" with future markets. Milán: Journal of the History of Economic Thought - Bocconi University.

Federación Nacional de Cafeteros de Colombia (2010). El árbol y el entorno. Recuperado de: http://www.cafedecolombia.com/particulares/es/sobre_el_cafe/el_cafe/ el_arbol_y_el_entorno/

Gibson, R. y Schwartz , E. S. (1990). Stochastic Convenience Yield and the Pricing of Oil Contigent Claims. Recuperado de: http://www.anderson.ucla.edu/documents/areas/ fac/finance/42.pdf

German, H. y Smith, W. O. (2012). Theory of Storage, Inventory and Volatility in the LME Base Metals. Recuperado de: http://epge.fgv.br/conferencias/commodity-prices/ files/HelyetteGeman.pdf

Helfrich, S. (2009). On the Modelling, Design and Valuation of Commodity Derivatives. Manchester: University of Manchester.

Hull, J. C. (2009). Options, futures, and other derivatives. Pearson - Prentice Hall.

Ibbotson Associates (2006, 27 de marzo). Strategic Asset Allocation and Commodities. Recuperado de: http://corporate.morningstar.com/ib/documents/MethodologyDocuments/IBBAssociates/Commodities.pdf

IntercontinentalExchange Inc. (2013). SPAN Margin System. Recuperado de: https://www. theice.com/publicdocs/clear_us/SPAN_Explanation.pdf

IntercontinentalExchange, Inc. (2013). Coffee ' $C$ '. Recuperado de: https://www.theice. com/publicdocs/ICE_Coffee_Brochure.pdf

International Trade Centre (2011 de febrero). The Coffee Guide. Recuperado de: http:// www.thecoffeeguide.org/coffee-guide/

Kellerhals, B. P. (2004). Asset Pricing Modeling and Estimation. Berlin: Springer. 
Laverde, G.F. (2011). Contratación de paradigmas de las finanzas: normalidad e hipótesis del mercado eficiente.Aplicaciones en Matlab. En Laverde, G.F. Observatorio de Economía y Operaciones Numéricas (pp. 172-175). Bogotá: Universidad Externado de Colombia.

Mejía, C. (2014). Administración del Riesgo Temporal de Liquidez asociado a los llamados al margen dentro del negocio de comercialización del café verde en Colombia. Bogotá: Universidad Externado de Colombia.

Organización Internacional del Café (2013). Informe mensual sobre el mercado del café. Londres: Organización Internacional del Café.

Pindyck, R. S. y Rubinfeld, D. L. (2006). Microeconomía. Madrid: Pearson - Prentice Hall.

Ribeiro, D. R. y Hodges, S. D. (2004, 11 de agosto). Equilibrium Model for Commodity Prices: Competitive and Monopolistic Markets. Recuperado de: http://www2. warwick.ac.uk/fac/soc/wbs/subjects/finance/research/wpaperseries/2004/04-203. pdfSchwartz, E. S. (1997, julio). The Stochastic Behavior of Commodity Prices: Implications for Valuation and Hedging. Recuperado de: http://www.anderson.ucla. edu/documents/areas/fac/finance/64.pdf

Smith, J. E. y Schwartz, E. (2000, julio). Short - Term Variations and Long - Term Dynamics in Commodity Prices. Recuperado de: http://www.anderson.ucla.edu/documents/ areas/fac/finance/72.pdf

Taulli, T. (2011). All About Commodities: The easy way to get started. New York: McGraw-Hill.

Thomas, E. W. (2012, abril). Obsolescence Risk and the Systematic Destruction of Wealth. Recuperado de: http://www.ermsymposium.org/2012/OtherPapers/Wendling-Paper-12-10-11.pdf

U.S. Department of the Treasury (2013, 26 de noviembre). Daily Treasury Yield Curve Rates. Recuperado de: http://www.treasury.gov/resource-center/data-chart-center/ interest-rates/Pages/TextView.aspx?data=yieldYear\&year=2010

Wilmott P. (2007). Quantitative Finance. Recuperado de: http://www.untag-smd.ac.id/ files/Perpustakaan_Digital_1/FINANCE\%20Paul\%20Wilmott\%20Introduces\%20 Quantitative\%20Finance\%200470319585.pdf

Zolotko, M. (2012). Modelling general dependence between commodity forward curves. Berlin: Economic Risk. 


\section{Anexo 1. El proceso de iteración de la cuenta de margen}

Una forma de representar la variación en la cuenta de margen para un portafolio de Contratos "C" de café de la ICE, en los que se tiene una posición corta, a través del tiempo, es la siguiente (Mejía, 2014):

$$
\left(\Delta M_{t}\right) x=\left(\frac{F_{t, T}-F_{t+\Delta t, T}}{\gamma}\right) m x
$$

\section{Donde}

$$
\Delta M_{t}=\left(M_{t+\Delta t}-M_{t}\right) x \text { es la variación en la cuenta de margen }
$$

Al resolver la Ecuación en Diferencias por el método de la iteración se tiene que:

$$
\begin{gathered}
\left(\Delta M_{t}\right) x=\left(\frac{F_{t, T}-F_{t+\Delta t, T}}{\gamma}\right) m x \\
\left(M_{t+\Delta t}-M_{t}\right) x=\left(\frac{F_{t, T}-F_{t+\Delta t, T}}{\gamma}\right) m x \\
M_{t+\Delta t} x=\left(\frac{F_{t, T}-F_{t+\Delta t, T}}{\gamma}\right) m x+M_{t} x
\end{gathered}
$$

Asumiendo que $\Delta t=1$ e iterando:

$$
\begin{gathered}
t=0 \\
M_{1}=\left(\frac{F_{0, T}-F_{1, T}}{\gamma}\right) m x+M_{0} x
\end{gathered}
$$




$$
\begin{gathered}
t=1 \\
M_{2}=\left(\frac{F_{1, T}-F_{2, T}}{\gamma}\right) m x+M_{1} x
\end{gathered}
$$

$$
\begin{gathered}
\text { Dado que } M_{1}=\left(\frac{F_{0, T}-F_{1, T}}{\gamma}\right) m x+M_{0} x \\
M_{2}=\left(\frac{F_{1, T}-F_{2, T}}{\gamma}\right) m x+\left[\left(\frac{F_{0, T}-F_{1, T}}{\gamma}\right) m x+M_{0} x\right] \\
M_{2}=\left(\frac{F_{1, T}-F_{2, T}}{\gamma}\right) m x+\left(\frac{F_{0, T}-F_{1, T}}{\gamma}\right) m x+M_{0} x \\
M_{2}=\left(\frac{F_{1, T}-F_{2, T}+F_{0, T}-F_{1, T}}{\gamma}\right) m x+M_{0} x \\
M_{2}=\left(\frac{F_{0, T}-F_{2, T}}{\gamma}\right) m x+M_{0} x \\
M_{3}=\left(\frac{F_{2, T}-F_{3, T}}{\gamma}\right) m x+M_{2}
\end{gathered}
$$

Dado que $M_{2}=\left(\frac{F_{0, T}-F_{2, T}}{\gamma}\right) m x+M_{0} x$ 


$$
\begin{gathered}
M_{3}=\left(\frac{F_{2, T}-F_{3, T}}{\gamma}\right) m x+\left[\left(\frac{F_{0, T}-F_{2, T}}{\gamma}\right) m x+M_{0} x\right] \\
M_{3}=\left(\frac{F_{2, T}-F_{3, T}}{\gamma}\right) m x+\left(\frac{F_{0, T}-F_{2, T}}{\gamma}\right) m x+M_{0} x \\
M_{3}=\left(\frac{F_{2, T}-F_{3, T}+F_{0, T}-F_{2, T}}{\gamma}\right) m x+M_{0} x \\
M_{3}=\left(\frac{F_{0, T}-F_{3, T}}{\gamma}\right) m x+M_{0} x
\end{gathered}
$$

Dado que $M_{0}=M_{I}$ y generalizando:

$$
M_{t}=\left(\frac{F_{0, T}-F_{t, T}}{\gamma}\right) m x+M_{I} x
$$

\section{Anexo 2. La estrategia del Cash \& Carry}

La ecuación 8 es correcta ya que adaptando las transacciones descritas por German (2005, citado por Helfrich, 2009), las oportunidades de arbitraje se anulan:

En el momento $t=0$ :

- Se endeuda a la Tasa Libre de Riesgo en un monto de $37.500 * S(0) e^{\delta T_{1}}$.

- Con esos recursos compra 37.500 de café verde estándar en el mercado físico internacional al Precio Spot Estándar $S(0)$.

- Toma una posición corta en el mercado financiero para entregar 37.500 libras de café verde estandarizado en el tiempo $t=T_{1}$ al precio $F\left(0, T_{1}\right)$. 
En el momento $t=T_{1}$ :

- Reinvierte la Convenience Yield Neta.

- Paga la deuda por un monto de $37.500 * S(0) \mathrm{e}^{\left(r_{f}-\delta\right) T_{1}}$.

- Entrega las 37.500 libras y recibe un pago por $37.500 * F\left(0, T_{1}\right)$.

El balance al final es el siguiente:

$$
\begin{gathered}
\text { Resultado }=-37.500 * S(0) e^{\delta T_{1}}+37.500 * S(0) e^{\delta T_{1}}-37.500 * S(0) e^{\left(r_{f}-\delta\right) T_{1}} \\
+37.500 * F\left(0, T_{1}\right) \\
\text { Resultado }=-37.500 *\left[-S(0) e^{\delta T_{1}}+S(0) e^{\delta T_{1}}-S(0) e^{\left(r_{f}-\delta\right) T_{1}}+F\left(0, T_{1}\right)\right] \\
\text { Resultado }=-37.500 *\left[F\left(0, T_{1}\right)-S(0) e^{(r-\delta) T_{1}}\right] \\
\text { Resultado }=0
\end{gathered}
$$

\section{Anexo 3. Dinámica del Precio Futuro del Contrato "C" y su solución parte I}

A partir de las ecuaciones 8 y 9:

$$
\begin{gathered}
F(t, T)=S(t) e^{\left(r_{f}-\delta\right)(T-t)} \\
d S(t)=\mu_{C P} S(t) d t+\sigma_{S} S(t) d W(t)
\end{gathered}
$$

Se puede encontrar la dinámica del Precio Futuro aplicando el lema de Ito (Hull, 2009). Se tiene la función $f(t, x)=x e^{\left(r_{f}-\delta\right)(T-t)}$ doblemente diferenciable. Se toman las derivadas parciales:

$$
\frac{\partial f(t, x)}{\partial t}=x e^{\left(r_{f}-\delta\right)(T-t)}\left(\delta-r_{f}\right)+0 * e^{\left(r_{f}-\delta\right)(T-t)}
$$




$$
\begin{aligned}
& \frac{\partial f(t, x)}{\partial t}=x e^{\left(r_{f}-\delta\right)(T-t)}\left(\delta-r_{f}\right) \\
& \frac{\partial f(t, x)}{\partial x}=1 e^{\left(r_{f}-\delta\right)(T-t)}+x * 0
\end{aligned}
$$

$$
\frac{\partial f(t, x)}{\partial x}=1 e^{\left(r_{f}-\delta\right)(T-t)}
$$

$$
\frac{\partial f(t, x)}{\partial x}=e^{\left(r_{f}-\delta\right)(T-t)}
$$

$$
\frac{\partial f^{2}(t, x)}{\partial x^{2}}=1 * 0+0 * e^{\left(r_{f}-\delta\right)(T-t)}
$$

$$
\frac{\partial f^{2}(t, x)}{\partial x^{2}}=0
$$

Aplicando el lema de Ito:

$$
\begin{aligned}
d f[t, S(t)]= & \left\{\frac{\partial f}{\partial t}+\mu[t, S(t)] \frac{\partial f}{\partial S(t)}+\frac{1}{2} \sigma^{2}[t, S(t)] \frac{\partial^{2} f}{\partial S(t)^{2}}\right\} d t+\sigma[t, S(t)] \frac{\partial f}{\partial S(t)} d W(t) \\
d f[t, S(t)]= & \left\{S(t) e^{\left(r_{f}-\delta\right)(T-t)}(\delta-r)+\left(\mu_{C P}-\delta\right) S(t) e^{\left(r_{f}-\delta\right)(T-t)}+\frac{1}{2}\left[\sigma_{S} S(t)\right]^{2} 0\right\} d t \\
& +\sigma_{S} S(t) e^{\left(r_{f}-\delta\right)(T-t)} d W(t)
\end{aligned}
$$




$$
\begin{gathered}
d f[t, S(t)]=\left\{S(t) e^{\left(r_{f}-\delta\right)(T-t)}(\delta-r)+\left(\mu_{C P}-\delta\right) S(t) e^{\left(r_{f}-\delta\right)(T-t)}\right\} d t \\
+\sigma_{S} S(t) e^{\left(r_{f}-\delta\right)(T-t)} d W(t) \\
\text { Dado que } F(t, T)=S(t) e^{\left(r_{f}-\delta\right)(T-t)} \\
d F(t, T)=\left\{F(t, T)\left(\delta-r_{f}\right)+\left(\mu_{C P}-\delta\right) F(t, T)\right\} d t+\sigma_{S} F(t, T) d W(t) \\
d F(t, T)=F(t, T)\left[\left(\delta-r_{f}\right)+\left(\mu_{C P}-\delta\right)\right] d t+\sigma_{S} F(t, T) d W(t) \\
d F(t, T)=F(t, T)\left(\mu_{C P}-r_{f}\right) d t+\sigma_{S} F(t, T) d W(t) \quad(10)
\end{gathered}
$$

\section{Anexo 4. Dinámica del Precio Futuro del Contrato "C" parte II}

Se procede a resolver la ecuación 10 nuevamente a través del lema de Ito. Se tiene la función $f(t, x)=\operatorname{Ln}(x)$ doblemente diferenciable. Se toman las derivadas parciales:

$$
\begin{gathered}
\frac{\partial f(t, x)}{\partial t}=0 \\
\frac{\partial f(t, x)}{\partial x}=\frac{1}{x} \\
\frac{\partial f^{2}(t, x)}{\partial x^{2}}=-\frac{1}{x^{2}}
\end{gathered}
$$


Aplicando el lema de Ito:

$$
\begin{gathered}
d f[t, F(t, T)]=\left\{\frac{\partial f}{\partial t}+\mu[t, S(t)] \frac{\partial f}{\partial F(t, T)}+\frac{1}{2} \sigma^{2}[t, S(t)] \frac{\partial^{2} f}{\partial S(t)^{2}}\right\} d t \\
+\sigma[t, S(t)] \frac{\partial f}{\partial S(t)} d W(t) \\
d \operatorname{Ln}[F(t, T)]=\left\{0+F(t, T)\left(\mu_{C P}-r_{f}\right) \frac{1}{F(t, T)}-\frac{1}{2}\left[\sigma_{S} F(t, T)\right]^{2} \frac{1}{F(t, T)^{2}}\right\} d t \\
+\sigma_{S} F(t, T) \frac{1}{F(t, T)} d W(t) \\
d \operatorname{Ln}[F(t, T)]=\left[\left(\mu_{C P}-r_{f}\right)-\frac{1}{2} \sigma_{S}^{2}\right] d t+\sigma_{S} d W(t)
\end{gathered}
$$

Integrando entre 0 y $t$$$
\operatorname{Ln}[F(t, T)]=\operatorname{Ln}[F(0, T)]+\int_{0}^{t}\left[\left(\mu_{C P}-r_{f}\right)-\frac{1}{2} \sigma_{S}^{2}\right]+\int_{0}^{t} \sigma_{S} d W(z)
$$$$
\operatorname{Ln}[F(t, T)]=\operatorname{Ln}[F(0, T)]+\left[\left(\mu_{C P}-r_{f}\right)-\frac{1}{2} \sigma_{S}^{2}\right] \int_{0}^{t} d t+\sigma_{S} \int_{0}^{t} d W(z)
$$$$
\operatorname{Ln}[F(t, T)]=\operatorname{Ln}[F(0, T)]+\left[\left(\mu_{C P}-r_{f}\right)-\frac{1}{2}{\sigma_{S}}^{2}\right] t+\sigma_{S} \int_{0}^{t} d W(z)
$$ 
Sacando el exponencial a ambos lados

$$
\begin{aligned}
& e^{L n[F(t, T)]}=e^{\operatorname{Ln}[F(0, T)]+\left[\left(\mu_{C P}-r_{f}\right)-\frac{1}{2} \sigma_{S}^{2}\right] t+\sigma_{S} \int_{0}^{t} d W(z)} \\
& e^{L n[F(t, T)]}=e^{L n[F(0, T)]} e^{\left[\left(\mu_{C P}-r_{f}\right)-\frac{1}{2} \sigma_{S}^{2}\right] t+\sigma_{S} \int_{0}^{t} d W(z)} \\
& F(t, T)=F(0, T) e^{\left[\left(\mu_{C P}-r_{f}\right)-\frac{1}{2} \sigma_{S}^{2}\right] t+\sigma_{S} \int_{0}^{t} d W(z)}
\end{aligned}
$$

\section{Anexo 5. Calibración del modelo asociado al escenario de backtesting}

Ahora bien, existen diversos métodos para calibrar (estimar los parámetros) del modelo presentado en la sección anterior. Sin embargo, como lo señala Schwartz (1997), uno de los problemas con la implementación empírica de los modelos del Precio Futuro sobre commodities es que frecuentemente los factores de los mismos - que para este caso corresponden al Precio Spot Estándar, la Convenience Yield Neta Instantánea y la Tasa Libre de Riesgo Instantánea- no son directamente observables.

\section{El Precio Futuro del Contrato "C"}

Dado el escenario planteado en el capítulo 2, el Precio Futuro $F_{t, T}$ se tomará, para cada día de interés, de la serie histórica del Precio Futuro del Contrato "C" con vencimiento en septiembre de 2010, desde el 19 de mayo hasta su vencimiento el 20 de septiembre de 2010 (Mejía, 2014).

\section{Aproximaciones al Precio Spot Estándar y al Retorno Simple Neto Instantáneo}

Una aproximación al Precio Spot Estándar deriva tanto de la lógica misma de los contratos futuros como de la forma como se manejan a la hora de realizar coberturas para mitigar el riesgo de precio (Mejía, 2014): 
- Por lo visto en la sección 1, el First Notice Day se constituye en la fecha a partir de la cual la ICE comienza a asignar las posiciones cortas con las largas abiertas para entregar el café verde. En ese sentido, el Precio Futuro posterior a $t=n$ se constituye en uno de entrega prácticamente inmediata.

- Por lo visto en la sección 2.1, dado que los contratos futuros se negocian hasta el First Notice Day, momento hasta el cual se puede cerrar la posición con el importador, la fecha para embarcar el café verde oscilará alrededor de $t=n$. Por ende, una aproximación al precio de pronta entrega serán aquellos que corresponden a algunos días previos y posteriores a esta fecha hasta el vencimiento en $t=T$.

De esta forma, si uno toma cada uno de estos precios y los junta para cada contrato futuro, se obtiene una serie continua del Precio Spot Estándar conocida como la Primera Posición del Contrato Genérico. Atendiendo a la lógica observada en la sección 1.1 , se tiene que su símbolo es $\mathrm{KC}^{159}$. Con base en lo anterior, y dado el escenario planteado en el capítulo 2, el Precio Spot Estándar $S_{t}$ se tomará de la serie histórica de la Primera Posición del Contrato Genérico del Contrato " $C$ " desde el 19 de mayo hasta el 20 de septiembre de 2010 (Mejía, 2014).

Ahora bien, para estimar la media total $\mu^{60}$ y la desviación estándar $\sigma$ de corto plazo del Retorno Simple Neto Instantáneo del Precio Spot Estándar para cada día, se tomará el promedio simple y la desviación estándar de los Retornos Simples Netos Diarios de la Primera Posición del Contrato Genérico desde la fecha de interés hasta el 20 de septiembre de 2010 (Mejía, 2014):

$$
\begin{gathered}
\mu_{t}=\frac{1}{n} \sum_{i=t}^{n} r_{i} \\
\sigma_{t}=\sqrt[2]{\frac{1}{n-1} \sum_{i=t}^{n}\left(r_{i}-\mu_{t}\right)^{2}}
\end{gathered}
$$

$59 \quad$ Este símbolo es también el Ticker en Bloomberg L. P. Si se unen los precios de la segunda posición relevante se obtiene la Segunda Posición del Contrato Genérico, cuyo símbolo es Kc2 y así sucesivamente.

60 Corresponde a la media del Retorno Simple Neto Instantáneo de corto plazo más la Convenience Yield Neta Instantánea $\delta$. 
Donde:

$\mu_{t}$ : media de los Retornos Simples Netos Diarios del Precio Spot Estándar en el tiempo $t$

$\sigma_{t}$ : desviación de los Retornos Simples Netos Diarios del Precio Spot Estándar en el tiempo $t$

$r_{i}$ : Retorno Simple Neto Diario en el tiempo $i$

$n$ : número de datos desde la fecha de interés hasta el vencimiento

En ese sentido, la media total diaria $\mu$ para el periodo comprendido entre el 19 de mayo y el 20 de septiembre de 2010 es del 0,3899\% con una desviación estándar diaria del 2,2563\%. Por otro lado, la evolución de estos parámetros va cambiando a medida que se va conociendo nueva información, como se aprecia en la tabla 6 (Mejía, 2014): 
Tabla 6. Medias y desviaciones estándar de la Primera Posición Genérica del Contrato " $C$ " para distintas fechas de interés

Cobertura 1

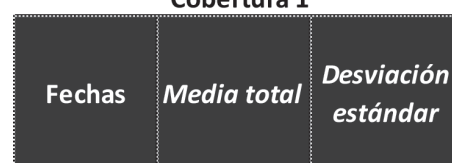

\begin{tabular}{|l|l|l|}
$19 / 05 / 2010$ & $0,3899 \%$ & $2,2563 \%$ \\
\hline
\end{tabular}

\begin{tabular}{|l|l|l|}
\hline $20 / 05 / 2010$ & $0,4022 \%$ & $2,2670 \%$ \\
\hline
\end{tabular}

\begin{tabular}{|l|l|l|}
\hline $21 / 05 / 2010$ & $0,4002 \%$ & $2,2807 \%$ \\
\hline
\end{tabular}

\begin{tabular}{|l|l|l|}
\hline $24 / 05 / 2010$ & $0,4064 \%$ & $2,2940 \%$ \\
\hline
\end{tabular}

\begin{tabular}{|l|l|l|}
\hline $25 / 05 / 2010$ & $0,4068 \%$ & $2,3083 \%$ \\
\hline
\end{tabular}

\begin{tabular}{|l|l|l|}
\hline $26 / 05 / 2010$ & $0,4029 \%$ & $2,3226 \%$ \\
\hline
\end{tabular}

\begin{tabular}{|l|l|l|}
\hline $27 / 05 / 2010$ & $0,3981 \%$ & $2,3370 \%$ \\
\hline
\end{tabular}

\begin{tabular}{|l|l|l|}
\hline $28 / 05 / 2010$ & $0,4079 \%$ & $2,3505 \%$ \\
\hline
\end{tabular}

\begin{tabular}{|l|l|l|}
\hline $01 / 06 / 2010$ & $0,3910 \%$ & $2,3611 \%$ \\
\hline
\end{tabular}

\begin{tabular}{|l|l|l|}
\hline $02 / 06 / 2010$ & $0,4072 \%$ & $2,3725 \%$ \\
\hline $03 / 06 / 2010$ & $0,4107 \%$ & $2,3883 \%$ \\
\hline
\end{tabular}

\begin{tabular}{|l|l|l|}
\hline $03 / 06 / 2010$ & $0,4107 \%$ & $2,3883 \%$ \\
\hline
\end{tabular}

\begin{tabular}{|l|l|l|}
\hline $04 / 06 / 2010$ & $0,4347 \%$ & $2,3955 \%$ \\
\hline
\end{tabular}

\begin{tabular}{|l|l|l|}
\hline $07 / 06 / 2010$ & $0,4473 \%$ & $2,4096 \%$ \\
\hline 0
\end{tabular}

\begin{tabular}{|l|l|l|}
\hline $08 / 06 / 2010$ & $0,4488 \%$ & $2,4264 \%$ \\
\hline
\end{tabular}

\begin{tabular}{|l|l|l|}
\hline $09 / 06 / 2010$ & $0,4456 \%$ & $2,4436 \%$ \\
\hline $10 / 06 / 2010$ & $0,4249 \%$ & $2,4549 \%$ \\
\hline
\end{tabular}

\begin{tabular}{|l|l|l|}
\hline $10 / 06 / 2010$ & $0,4249 \%$ & $2,4549 \%$ \\
\hline
\end{tabular}

\begin{tabular}{|l|l|l|}
\hline $11 / 06 / 2010$ & $0,3469 \%$ & $2,3840 \%$ \\
\hline $14 / 06 / 2010$
\end{tabular}

\begin{tabular}{|l|l|l|}
\hline $14 / 06 / 2010$ & $0,2912 \%$ & $2,3560 \%$ \\
\hline
\end{tabular}

\begin{tabular}{|l|l|l|}
\hline $15 / 06 / 2010$ & $0,2125 \%$ & $2,2819 \%$ \\
\hline
\end{tabular}

\begin{tabular}{|l|l|l|}
\hline $16 / 06 / 2010$ & $0,2214 \%$ & $2,2982 \%$ \\
\hline $17 / 06 / 2010$
\end{tabular}

\begin{tabular}{|l|l|l|}
\hline $17 / 06 / 2010$ & $0,2481 \%$ & $2,3058 \%$ \\
\hline
\end{tabular}

\begin{tabular}{|l|l|l|}
\hline $18 / 06 / 2010$ & $0,2130 \%$ & $2,3064 \%$ \\
\hline
\end{tabular}

\begin{tabular}{|l|l|l}
$21 / 06 / 2010$ & $0,2525 \%$ & $2,3030 \%$ \\
\hline
\end{tabular}

\begin{tabular}{|l|l|l|}
\hline $22 / 06 / 2010$ & $0,2308 \%$ & $2,3153 \%$ \\
\hline
\end{tabular}

\begin{tabular}{|l|l|l}
\hline $23 / 06 / 2010$ & $0,2397 \%$ & $2,3335 \%$ \\
\hline
\end{tabular}

\begin{tabular}{|l|l|l|}
\hline $24 / 06 / 2010$ & $0,1581 \%$ & $2,2636 \%$ \\
\hline
\end{tabular}

\begin{tabular}{|l|l|l|}
\hline $25 / 06 / 2010$ & $0,1582 \%$ & $2,2830 \%$ \\
\hline
\end{tabular}

\begin{tabular}{|l|l|l|}
\hline $28 / 06 / 2010$ & $0,1682 \%$ & $2,3017 \%$ \\
\hline
\end{tabular}

\begin{tabular}{|l|l|l}
$29 / 06 / 2010$ & $0,2218 \%$ & $2,2853 \%$ \\
\hline
\end{tabular}

\begin{tabular}{|l|l|l}
$30 / 06 / 2010$ & $0,1959 \%$ & $2,2975 \%$ \\
\hline
\end{tabular}

\begin{tabular}{|l|l|l|}
\hline $01 / 07 / 2010$ & $0,1773 \%$ & $2,3145 \%$ \\
\hline
\end{tabular}

\begin{tabular}{|l|l|l|}
\hline $02 / 07 / 2010$ & $0,2240 \%$ & $2,3099 \%$ \\
\hline
\end{tabular}

\begin{tabular}{|l|l|l|}
\hline $06 / 07 / 2010$ & $0,3073 \%$ & $2,2486 \%$ \\
\hline $07 / 07 / 2010$ & $0,2495 \%$ & $2,2305 \%$ \\
\hline
\end{tabular}

Tomado de Mejía (2014). 


\section{Aproximaciones a la Tasa Libre de Riesgo y la Convenience Yield Neta}

Dado que el Contrato "C" se negocia en centavos de dólar por libra de Estados Unidos y se transa en Nueva York, se tomará como aproximación a la Tasa Libre de Riesgo Instantánea la tasa vencida periódica trimestral de los bonos del Tesoro norteamericano con vencimiento a tres meses ${ }^{61}$ (U.S. Department of the Treasury, 2013). Ahora bien, a partir de la ecuación 4 se puede estimar la Convenience Yield Neta Diaria como aproximación de la Convenience Yield Neta Instantánea de la siguiente forma (Schwartz, 1997):

$$
\begin{gathered}
F(0, T)=S(0) e^{\left(r_{f}-\delta\right)\left(T_{1}-0\right)} \\
\frac{F(0, T)}{S(0)}=e^{\left(r_{f}-\delta\right)\left(T_{1}-0\right)} \\
\operatorname{Ln}\left[\frac{F(0, T)}{S(0)}\right]=\left(r_{f}-\delta\right)\left(T_{1}-0\right) \\
\frac{\operatorname{Ln}\left[\frac{F(0, T)}{S(0)}\right]}{(T-0)}=\left(r_{f}-\delta\right) \\
\frac{\operatorname{Ln}[F(0, T)]-\operatorname{Ln}[S(0)]}{\left(T_{1}-0\right)}=\left(r_{f}-\delta\right) \\
r_{f}-\frac{\operatorname{Ln}[F(0, T)]-\operatorname{Ln}[S(0)]}{(T-0)}=\delta
\end{gathered}
$$

61 La selección de la Tasa Libre de Riesgo se hizo tomando en cuenta que la vida de los flujos es de alrededor de tres meses, en cuanto se trabaja con la Primera Posición Relevante o la Primera Posición Activa. Adicionalmente, se sigue a Ross (2002) quien se inclina por el uso de los T-Bills de 90 días de duración (Bravo Orellana, s. f.). 
Con base en lo anterior, para el 19 de mayo de 2010, dado el Precio Spot Estándar de $S_{0}=132,50$, el Precio Futuro del Contrato "C" con vencimiento el 20 de septiembre de 2010 de $F_{0, T}=133,80$ y la Tasa Libre de Riesgo Diaria $r_{f}=0,0019 \%{ }^{62}$, la Convenience Yield Neta Diaria es igual a (Mejía, 2014):

$$
\begin{gathered}
0,0019 \%-\frac{\operatorname{Ln}(133,80)-\operatorname{Ln}(132,50)}{86}=\delta \\
-0,0095 \%=\delta
\end{gathered}
$$

$\mathrm{Al}$ realizar este mismo procedimiento para todas las fechas se obtiene la tabla 7 (Mejía, 2014):

62 Para transformar la Tasa Libre de Riesgo, se toma la vencida trimestre periódica, se multiplica por cuatro para pasarla a nominal trimestre vencida y, posteriormente, se pasa a efectiva anual. De ahí, se pasa a nominal diaria vencida (con base 365) y se divide en 365 para tener la vencida diaria periódica. Siguiendo a Hull (2009), esta periodicidad es un excelente proxy de la tasa continua. 
Tabla 7. Variables por utilizar en el escenario de backtesting

\begin{tabular}{|c|c|c|c|c|c|c|c|c|c|c|}
\hline Fechas & $\begin{array}{c}\text { Precio } \\
\text { Spot } \\
\text { Estándar }\end{array}$ & $\begin{array}{l}\text { Precio } \\
\text { Futuro }\end{array}$ & $\begin{array}{l}\text { Tasa Libre } \\
\text { de Riesgo } \\
\text { trimestre } \\
\text { periódica }\end{array}$ & $\begin{array}{c}\text { Tasa Libre } \\
\text { de Riesgo } \\
\text { E.A. }\end{array}$ & \begin{tabular}{|c} 
Tasa Libre de \\
Riesgo \\
diaria \\
periódica
\end{tabular} & $\begin{array}{l}\text { Convenience } \\
\text { Yield Neta } \\
\text { diaria } \\
\text { períodica }\end{array}$ & $\begin{array}{l}\text { Días hasta el } \\
\text { vencimiento }\end{array}$ & Media total & Media & $\begin{array}{l}\text { Desviación } \\
\text { estándar }\end{array}$ \\
\hline $19 / 05 / 2010$ & 132,50 & 133,80 & $0,1700 \%$ & $0,6817 \%$ & $0,0019 \%$ & $-0,0095 \%$ & 86 & $0,3899 \%$ & $0,3804 \%$ & $2,2563 \%$ \\
\hline $20 / 05 / 2010$ & 131,65 & 132,95 & $0,1700 \%$ & $0,6817 \%$ & $0,0019 \%$ & $-0,0097 \%$ & 85 & $0,4022 \%$ & $0,3925 \%$ & $2,2670 \%$ \\
\hline $21 / 05 / 2010$ & 132,40 & 133,70 & $0,1700 \%$ & $0,6817 \%$ & $0,0019 \%$ & $-0,0098 \%$ & 84 & $0,4002 \%$ & $0,3904 \%$ & $2,2807 \%$ \\
\hline $24 / 05 / 2010$ & 132,25 & 133,65 & $0,1700 \%$ & $0,6817 \%$ & $0,0019 \%$ & $-0,0108 \%$ & 83 & $0,4064 \%$ & $0,3956 \%$ & $2,2940 \%$ \\
\hline $25 / 05 / 2010$ & 132,75 & 134,25 & $0,1700 \%$ & $0,6817 \%$ & $0,0019 \%$ & $-0,0118 \%$ & 82 & $0,4068 \%$ & $0,3949 \%$ & $2,3083 \%$ \\
\hline $26 / 05 / 2010$ & 133,70 & 135,25 & $0,1700 \%$ & $0,6817 \%$ & $0,0019 \%$ & $-0,0124 \%$ & 81 & $0,4029 \%$ & $0,3906 \%$ & $2,3226 \%$ \\
\hline $27 / 05 / 2010$ & 134,75 & 136,30 & $0,1700 \%$ & $0,6817 \%$ & $0,0019 \%$ & $-0,0124 \%$ & 80 & $0,3981 \%$ & $0,3856 \%$ & $2,3370 \%$ \\
\hline $28 / 05 / 2010$ & 134,25 & 135,80 & $0,1600 \%$ & $0,6415 \%$ & $0,0018 \%$ & $-0,0128 \%$ & 79 & $0,4079 \%$ & $0,3952 \%$ & $2,3505 \%$ \\
\hline $01 / 06 / 2010$ & 136,55 & 138,05 & $0,1600 \%$ & $0,6415 \%$ & $0,0018 \%$ & $-0,0123 \%$ & 78 & $0,3910 \%$ & $0,3787 \%$ & $2,3611 \%$ \\
\hline $02 / 06 / 2010$ & 135,40 & 136,90 & $0,1600 \%$ & $0,6415 \%$ & $0,0018 \%$ & $-0,0126 \%$ & 77 & $0,4072 \%$ & $0,3947 \%$ & $2,3725 \%$ \\
\hline $03 / 06 / 2010$ & 135,60 & 137,05 & $0,1400 \%$ & $0,5612 \%$ & $0,0015 \%$ & $-0,0125 \%$ & 76 & $0,4107 \%$ & $0,3982 \%$ & $2,3883 \%$ \\
\hline $04 / 06 / 2010$ & 133,75 & 135,30 & $0,1400 \%$ & $0,5612 \%$ & $0,0015 \%$ & $-0,0138 \%$ & 75 & $0,4347 \%$ & $0,4208 \%$ & $2,3955 \%$ \\
\hline $07 / 06 / 2010$ & 133,10 & 134,60 & $0,1200 \%$ & $0,4809 \%$ & $0,0013 \%$ & $-0,0138 \%$ & 74 & $0,4473 \%$ & $0,4334 \%$ & $2,4096 \%$ \\
\hline $08 / 06 / 2010$ & 133,55 & 135,10 & $0,1200 \%$ & $0,4809 \%$ & $0,0013 \%$ & $-0,0145 \%$ & 73 & $0,4488 \%$ & $0,4343 \%$ & $2,4264 \%$ \\
\hline $09 / 06 / 2010$ & 134,45 & 136,05 & $0,1000 \%$ & $0,4006 \%$ & $0,0011 \%$ & $-0,0153 \%$ & 72 & $0,4456 \%$ & $0,4303 \%$ & $2,4436 \%$ \\
\hline $10 / 06 / 2010$ & 137,00 & 138,55 & $0,1000 \%$ & $0,4006 \%$ & $0,0011 \%$ & $-0,0148 \%$ & 71 & $0,4249 \%$ & $0,4101 \%$ & $2,4549 \%$ \\
\hline $11 / 06 / 2010$ & 144,95 & 146,10 & $0,0800 \%$ & $0,3204 \%$ & $0,0009 \%$ & $-0,0104 \%$ & 70 & $0,3469 \%$ & $0,3365 \%$ & $2,3840 \%$ \\
\hline $14 / 06 / 2010$ & 150,95 & 151,60 & $0,0700 \%$ & $0,2803 \%$ & $0,0008 \%$ & $-0,0055 \%$ & 69 & $0,2912 \%$ & $0,2857 \%$ & $2,3560 \%$ \\
\hline $15 / 06 / 2010$ & 159,35 & 159,95 & $0,0900 \%$ & $0,3605 \%$ & $0,0010 \%$ & $-0,0045 \%$ & 68 & $0,2125 \%$ & $0,2079 \%$ & $2,2819 \%$ \\
\hline $16 / 06 / 2010$ & 158,75 & 159,60 & $0,1000 \%$ & $0,4006 \%$ & $0,0011 \%$ & $-0,0069 \%$ & 67 & $0,2214 \%$ & $0,2145 \%$ & $2,2982 \%$ \\
\hline $17 / 06 / 2010$ & 156,35 & 157,80 & $0,0900 \%$ & $0,3605 \%$ & $0,0010 \%$ & $-0,0130 \%$ & 66 & $0,2481 \%$ & $0,2351 \%$ & $2,3058 \%$ \\
\hline $18 / 06 / 2010$ & 160,25 & 162,10 & $0,1100 \%$ & $0,4407 \%$ & $0,0012 \%$ & $-0,0165 \%$ & 65 & $0,2130 \%$ & $0,1965 \%$ & $2,3064 \%$ \\
\hline $21 / 06 / 2010$ & 156,60 & 160,80 & $0,1200 \%$ & $0,4809 \%$ & $0,0013 \%$ & $-0,0400 \%$ & 64 & $0,2525 \%$ & $0,2124 \%$ & $2,3030 \%$ \\
\hline $22 / 06 / 2010$ & 159,10 & 160,75 & $0,1300 \%$ & $0,5210 \%$ & $0,0014 \%$ & $-0,0150 \%$ & 63 & $0,2308 \%$ & $0,2159 \%$ & $2,3153 \%$ \\
\hline $23 / 06 / 2010$ & 158,60 & 160,50 & $0,1300 \%$ & $0,5210 \%$ & $0,0014 \%$ & $-0,0178 \%$ & 62 & $0,2397 \%$ & $0,2220 \%$ & $2,3335 \%$ \\
\hline $24 / 06 / 2010$ & 166,75 & 168,75 & $0,1300 \%$ & $0,5210 \%$ & $0,0014 \%$ & $-0,0181 \%$ & 61 & $0,1581 \%$ & $0,1400 \%$ & $2,2636 \%$ \\
\hline $25 / 06 / 2010$ & 167,00 & 168,90 & $0,1300 \%$ & $0,5210 \%$ & $0,0014 \%$ & $-0,0174 \%$ & 60 & $0,1582 \%$ & $0,1408 \%$ & $2,2830 \%$ \\
\hline $28 / 06 / 2010$ & 166,30 & 168,10 & $0,1700 \%$ & $0,6817 \%$ & $0,0019 \%$ & $-0,0164 \%$ & 59 & $0,1682 \%$ & $0,1518 \%$ & $2,3017 \%$ \\
\hline $29 / 06 / 2010$ & 161,50 & 163,15 & $0,1500 \%$ & $0,6014 \%$ & $0,0016 \%$ & $-0,0159 \%$ & 58 & $0,2218 \%$ & $0,2059 \%$ & $2,2853 \%$ \\
\hline $30 / 06 / 2010$ & 164,20 & 165,85 & $0,1800 \%$ & $0,7219 \%$ & $0,0020 \%$ & $-0,0156 \%$ & 57 & $0,1959 \%$ & $0,1803 \%$ & $2,2975 \%$ \\
\hline $01 / 07 / 2010$ & 166,20 & 168,25 & $0,1700 \%$ & $0,6817 \%$ & $0,0019 \%$ & $-0,0200 \%$ & 56 & $0,1773 \%$ & $0,1573 \%$ & $2,3145 \%$ \\
\hline $02 / 07 / 2010$ & 162,30 & 164,30 & $0,1700 \%$ & $0,6817 \%$ & $0,0019 \%$ & $-0,0204 \%$ & 55 & $0,2240 \%$ & $0,2036 \%$ & $2,3099 \%$ \\
\hline $06 / 07 / 2010$ & 155,50 & 157,65 & $0,1700 \%$ & $0,6817 \%$ & $0,0019 \%$ & $-0,0236 \%$ & 54 & $0,3073 \%$ & $0,2838 \%$ & $2,2486 \%$ \\
\hline $07 / 07 / 2010$ & 160,65 & 163,05 & $0,1600 \%$ & $0,6415 \%$ & $0,0018 \%$ & $-0,0262 \%$ & 53 & $0,2495 \%$ & $0,2233 \%$ & $2,2305 \%$ \\
\hline
\end{tabular}

Tomado de Mejía (2014). 